\title{
Second order asymptotic comparison of the MLE and MCLE for a two-sided truncated exponential family of distributions
}

\author{
M. Akahira ${ }^{1}$, S. Hashimoto ${ }^{2}$, K. Koike ${ }^{1}$, N. Ohyauchi ${ }^{1}$ \\ ${ }^{1}$ Institute of Mathematics, University of Tsukuba, Ibaraki, Japan \\ ${ }^{2}$ Graduate School of Pure and Applied Sciences, University of Tsukuba, \\ Ibaraki, Japan
}

\begin{abstract}
For a one-sided truncated exponential family of distributions with a natural parameter $\theta$ and a truncation parameter $\gamma$ as a nuisance parameter, it is shown by Akahira (2013) that the second order asymptotic loss of a bias-adjusted maximum likelihood estimator (MLE) $\hat{\theta}_{M L}^{*}$ of $\theta$ for unknown $\gamma$ relative to the MLE $\hat{\theta}_{M L}^{\gamma}$ of $\theta$ for known $\gamma$ is given and $\hat{\theta}_{M L}^{*}$ and the maximum conditional likelihood estimator (MCLE) $\hat{\theta}_{M C L}$ are second order asymptotically equivalent. In this paper, in a similar way to Akahira (2013), for a two-sided truncated exponential family of distributions with a natural parameter $\theta$ and two truncation parameters $\gamma$ and $\nu$ as nuisance ones, the stochastic expansions of the MLE $\hat{\theta}_{M L}^{\gamma, \nu}$ of $\theta$ for known $\gamma$ and $\nu$, the MLE $\hat{\theta}_{M L}$ and the MCLE $\hat{\theta}_{M C L}$ of $\theta$ for unknown $\gamma$ and $\nu$ are derived, their second order asymptotic means and variances are given, a bias-adjusted MLE $\hat{\theta}_{M L}^{*}$ and $\hat{\theta}_{M C L}$ are shown to be second order asymptotically equivalent, and the second order asymptotic losses of $\hat{\theta}_{M L}^{*}$ and $\hat{\theta}_{M C L}$ relative to $\hat{\theta}_{M L}^{\gamma, \nu}$ is also obtained. Further, some examples including an upper-truncated Pareto case are given.
\end{abstract}

Keywords: Natural parameter; Truncation parameter; Stochastic expansion; Maximum likelihood estimator; Maximum conditional likelihood estimator; Asymptotic variance; Second order asymptotic loss; Upper-truncated Pareto distribution

Mathematics Subject Classification: Primary 62F10; Secondary 62F12.

\section{Introduction}

The higher order asymptotic estimation in the presence of nuisance parameters is discussed by Akahira and Takeuchi (1982) in the pooled sample case and by Akahira and Takeuchi (1991) and 
Akahira (1997) in the sequential case, under suitable regularity conditions. For a truncated exponential family of distributions with a natural parameter $\theta$ and a truncation parameter $\gamma$ as a nuisance parameter which is regarded as a typical non-regular case, it is shown by Bar-Lev (1984) that the maximum likelihood estimator (MLE) $\hat{\theta}_{M L}^{\gamma}$ of $\theta$ for known $\gamma$ and the MLE and the maximum conditional likelihood estimator (MCLE) $\hat{\theta}_{M C L}$ of $\theta$ for unknown $\gamma$ have the same asymptotic normality (see, e.g. Andersen (1970) for the MCLE itself). A similar result can be derived from the stochastic expansions of $\hat{\theta}_{M L}^{\gamma}$ and $\hat{\theta}_{M L}$ in Akahira and Ohyauchi (2012). Hence, their estimators are seen to be asymptotically equivalent in the first order and asymptotically efficient. But it seems to be natural that $\hat{\theta}_{M L}$ and $\hat{\theta}_{M C L}$ for unknown $\gamma$ are asymptotically worse than $\hat{\theta}_{M L}^{\gamma}$ for known $\gamma$ in the higher order, since $\hat{\theta}_{M L}^{\gamma}$ has the full information on $\gamma$. In order to discriminate asymptotically efficient estimators, the concept of asymptotic deficiency discussed by Hodges and Lehmann (1970) takes an important role. Indeed, for two estimators $\hat{\theta}_{n}^{(1)}$ and $\hat{\theta}_{n}^{(2)}$ of $\theta$ based on a sample of size $n$, let $d_{n}$ be an additional size of sample needed such that $\hat{\theta}_{n}^{(2)}$ is asymptotically equivalent to $\hat{\theta}_{n}^{(1)}$ in some sense. If $\lim _{n \rightarrow \infty} d_{n}$ exists, it is called the asymptotic deficiency of $\hat{\theta}_{n}^{(2)}$ relative to $\hat{\theta}_{n}^{(1)}$, which is useful in comparing asymptotically efficient estimators up to the higher order and investigated by Akahira (1981, 1986, 1992) from the viewpoint of the equivalence of the asymptotic distributions of estimators up to the higher order, under suitable regularity conditions. For example, the asymptotic deficiency is shown to be closely related to the difference between the second order asymptotic variances of estimators. In Akahira (2013), the second order asymptotic losses of $\hat{\theta}_{M L}^{*}$ and $\hat{\theta}_{M C L}$ relative to $\hat{\theta}_{M L}^{\gamma}$ which correspond to the asymptotic deficiencies are obtained from their second order asymptotic variances which are calculated from their stochastic expansions. It is also shown that a bias-adjusted MLE $\hat{\theta}_{M L}^{*}$ and $\hat{\theta}_{M C L}$ of $\theta$ for unknown $\gamma$ are second order asymptotically equivalent and second order asymptotically worse than $\hat{\theta}_{M L}^{\gamma}$ of $\theta$ for known $\gamma$. On the other hand, for an upper-truncated Pareto distribution with an index parameter $\alpha$ to be estimated and two truncation parameters $\gamma$ and $\nu$ as nuisance ones, the MLE $\tilde{\alpha}$ of $\alpha$ for known $\gamma$ and $\nu$ and the MLE $\hat{\alpha}$ of $\alpha$ for unknown $\gamma$ and $\nu$ are shown to have the asymptotic normality by Aban et al. (2006). But, the distribution does not belong to the one-sided truncated exponential family discussed by Akahira (2013).

In this paper, in a similar way to Akahira (2013), the second order asymptotic results are extended to the case of a two-sided truncated exponential family of distributions with a natural parameter $\theta$ and two truncation parameters $\gamma$ and $\nu$ as nuisance ones, which includes the uppertruncated Pareto distribution. In Section 2, the formulation and assumptions are given. In Sections 
3 to 5 , the stochastic expansions of the MLE $\hat{\theta}_{M L}^{\gamma, \nu}$ of $\theta$ for known $\gamma$ and $\nu$, the MLE $\hat{\theta}_{M L}$ and the MCLE $\hat{\theta}_{M C L}$ of $\theta$ for unknown $\gamma$ and $\nu$ are derived, from which the second order asymptotic means and variances are obtained. In Section 6, the results in previous sections are summarized, the second order asymptotic losses of $\hat{\theta}_{M L}^{*}$ and $\hat{\theta}_{M C L}$ relative to $\hat{\theta}_{M L}^{\gamma, \nu}$ is obtained from their second order asymptotic variances, and a bias-adjusted MLE $\hat{\theta}_{M L}^{*}$ and $\hat{\theta}_{M C L}$ of $\theta$ for unknown $\gamma$ and $\nu$ are also shown to be second order asymptotically equivalent and second order asymptotically worse than the MLE $\hat{\theta}_{M L}^{\gamma, \nu}$ for known $\gamma$ and $\nu$. In Section 7, some examples on a two-sided truncated exponential distribution, an upper-truncated Pareto distribution and a two-sided truncated normal distribution are given.

\section{Formulation and assumptions}

In a similar way to Bar-Lev (1984) and Akahira (2013), we have the formulation as follows. Suppose that $X_{1}, X_{2}, \ldots, X_{n}, \ldots$ is a sequence of independent and identically distributed (i.i.d.) random variables with a density

$$
f(x ; \theta, \gamma, \nu)= \begin{cases}\frac{a(x) e^{\theta u(x)}}{b(\theta, \gamma, \nu)} & \text { for } c<\gamma \leq x \leq \nu<d \\ 0 & \text { otherwise }\end{cases}
$$

with respect to the Lebesgue measure, where $-\infty \leq c<d \leq \infty, a(\cdot)$ is a nonnegative-valued and continuous almost surely, and $u(\cdot)$ is absolutely continuous with $d u(x) / d x \not \equiv 0$ over the interval $(\gamma, \nu)$ for $\gamma, \nu \in(c, d)$ and $\gamma<\nu$. Let

$$
\Theta(\gamma, \nu):=\left\{\theta \mid 0<b(\theta, \gamma, \nu):=\int_{\gamma}^{\nu} a(x) e^{\theta u(x)} d x<\infty\right\}
$$

for $\gamma, \nu \in(c, d)$ and $\gamma<\nu$. Assume that for any $\gamma, \nu \in(c, d)$ with $\gamma<\nu, \Theta \equiv \Theta(\gamma, \nu)$ is a non-empty open interval. A family $\mathcal{P}:=\left\{P_{\theta, \gamma, \nu} \mid \theta \in \Theta, \gamma, \nu \in(c, d), \gamma<\nu\right\}$ of distributions $P_{\theta, \gamma, \nu}$ with (2.1) with a natural parameter $\theta$ and truncation parameters $\gamma, \nu$ is called a two-sided truncated exponential family of distributions. Then we consider the estimation problem on the natural parameter $\theta$ in the presence of nuisance parameters $\gamma$ and $\nu$.

In Akahira (2013), for a one-sided truncated exponential family of distributions with a natural parameter $\theta$ and a truncation parameter $\gamma$, the stochastic expansions of the MLE $\hat{\theta}_{M L}^{\gamma}$ of $\theta$ for known

$\gamma$, the MLE $\hat{\theta}_{M L}$ and MCLE $\hat{\theta}_{M C L}$ of $\theta$ for unknown $\gamma$ are derived and their estimators are compared up to the second order in their asymptotic variances, and a bias-adjusted MLE $\hat{\theta}_{M L}^{*}$ and $\hat{\theta}_{M C L}$ are 
shown to be second order asymptotically equivalent, but they are shown to be asymptotically worse than $\hat{\theta}_{M L}^{\gamma}$ in the second order, and the second order asymptotic losses on the asymptotic variances are obtained.

In a similar way to Akahira (2013), we extend the above results in Akahira (2013) to the case of a two-sided truncated exponential family of distributions with a natural parameter $\theta$ and two truncation parameters $\gamma$ and $\nu$. Henceforth, we derive the stochastic expansions of $\hat{\theta}_{M L}^{\gamma, \nu}, \hat{\theta}_{M L}$ and $\hat{\theta}_{M C L}$ up to the second order, i.e. the order $o_{p}\left(n^{-1}\right)$, get their asymptotic means and variances and obtain the second order asymptotic losses from the asymptotic variances.

\section{The stochastic expansion and the asymptotic variance of the MLE $\hat{\theta}_{M L}^{\gamma, \nu}$ of $\theta$ up to the second order when $\gamma$ and $\nu$ are known}

Denote a random vector $\left(X_{1}, \ldots, X_{n}\right)$ by $\boldsymbol{X}$, and let $X_{(1)} \leq \cdots \leq X_{(n)}$ be the corresponding order statistics of a random vector $\boldsymbol{X}$. Here we consider the case when $\gamma$ and $\nu$ are known. Then the density (2.1) is considered to belong to a regular exponential family of distributions with a natural parameter $\theta$, hence $\log b(\theta, \gamma, \nu)$ is strictly convex and infinitely differentiable in $\theta \in \Theta$ and

$$
\lambda_{k}(\theta, \gamma, \nu):=\frac{\partial^{k}}{\partial \theta^{k}} \log b(\theta, \gamma, \nu)
$$

is the $k$-th cumulant corresponding to $(2.1)$ for $k=1,2, \ldots$ For given $\boldsymbol{x}=\left(x_{1}, \ldots, x_{n}\right)$ satisfying $c<\gamma<x_{(1)}:=\min _{1 \leq i \leq n} x_{i}$ and $x_{(n)}:=\max _{1 \leq i \leq n} x_{i}<\nu<d$, the likelihood function of $\theta$ is given by

$$
L^{\gamma, \nu}(\theta ; \boldsymbol{x}):=\frac{1}{b^{n}(\theta, \gamma, \nu)}\left\{\prod_{i=1}^{n} a\left(x_{i}\right)\right\} \exp \left\{\theta \sum_{i=1}^{n} u\left(x_{i}\right)\right\} .
$$

Then the likelihood equation is

$$
\frac{1}{n} \sum_{i=1}^{n} u\left(x_{i}\right)-\lambda_{1}(\theta, \gamma, \nu)=0
$$

Since there exists a unique solution on $\theta$ of (3.2), we denote it by $\hat{\theta}_{M L}^{\gamma, \nu}$ which is the MLE of $\theta$ (see, e.g. Barndorff-Nielsen (1978) and Bar-Lev (1984)). In a similar way to Akahira (2013), we have 
from (3.2)

$$
\begin{aligned}
0= & \frac{1}{n} \sum_{i=1}^{n} u\left(X_{i}\right)-\lambda_{1}\left(\hat{\theta}_{M L}^{\gamma, \nu}, \gamma, \nu\right) \\
= & \frac{1}{n} \sum_{i=1}^{n}\left\{u\left(X_{i}\right)-\lambda_{1}(\theta, \gamma, \nu)\right\}-\frac{1}{\sqrt{n}} \lambda_{2}(\theta, \gamma, \nu) \sqrt{n}\left(\hat{\theta}_{M L}^{\gamma, \nu}-\theta\right)-\frac{1}{2 n} \lambda_{3}(\theta, \gamma, \nu) n\left(\hat{\theta}_{M L}^{\gamma, \nu}-\theta\right)^{2} \\
& -\frac{1}{6 n \sqrt{n}} \lambda_{4}(\theta, \gamma, \nu) n \sqrt{n}\left(\hat{\theta}_{M L}^{\gamma, \nu}-\theta\right)^{3}+O_{p}\left(\frac{1}{n^{2}}\right) .
\end{aligned}
$$

Putting

$$
\begin{aligned}
Z_{1} & :=\frac{1}{\sqrt{\lambda_{2}(\theta, \gamma, \nu) n}} \sum_{i=1}^{n}\left\{u\left(X_{i}\right)-\lambda_{1}(\theta, \gamma, \nu)\right\}, \\
U_{\gamma, \nu} & :=\sqrt{\lambda_{2}(\theta, \gamma, \nu) n}\left(\hat{\theta}_{M L}^{\gamma, \nu}-\theta\right),
\end{aligned}
$$

we obtain from (3.3)

$$
0=\sqrt{\frac{\lambda_{2}}{n}} Z_{1}-\sqrt{\frac{\lambda_{2}}{n}} U_{\gamma, \nu}-\frac{\lambda_{3}}{2 \lambda_{2} n} U_{\gamma, \nu}^{2}-\frac{\lambda_{4}}{6 \lambda_{2}^{3 / 2} n \sqrt{n}} U_{\gamma, \nu}^{3}+O_{p}\left(\frac{1}{n^{2}}\right),
$$

which implies that the stochastic expansion of $U_{\gamma, \nu}$ is given by

$$
U_{\gamma, \nu}=Z_{1}-\frac{\lambda_{3}}{2 \lambda_{2}^{3 / 2} \sqrt{n}} Z_{1}^{2}+\frac{1}{2 n}\left(\frac{\lambda_{3}^{2}}{\lambda_{2}^{3}}-\frac{\lambda_{4}}{3 \lambda_{2}^{2}}\right) Z_{1}^{3}+O_{p}\left(\frac{1}{n \sqrt{n}}\right),
$$

where $\lambda_{i}=\lambda_{i}(\theta, \gamma, \nu)(i=2,3,4)$. Since

$$
\begin{aligned}
& E_{\theta}\left(Z_{1}\right)=0, \quad V_{\theta}\left(Z_{1}\right)=E_{\theta}\left(Z_{1}^{2}\right)=1 \\
& E_{\theta}\left(Z_{1}^{3}\right)=\frac{\lambda_{3}}{\lambda_{2}^{3 / 2} \sqrt{n}}, \quad E_{\theta}\left(Z_{1}^{4}\right)=3+\frac{\lambda_{4}}{\lambda_{2}^{2} n},
\end{aligned}
$$

it follows that

$$
E_{\theta}\left(U_{\gamma, \nu}^{2}\right)=1+\left(\frac{11 \lambda_{3}^{2}}{4 \lambda_{2}^{3}}-\frac{\lambda_{4}}{\lambda_{2}^{2}}\right) \frac{1}{n}+O\left(\frac{1}{n \sqrt{n}}\right)
$$

Since

$$
E_{\theta}\left(U_{\gamma, \nu}\right)=-\frac{\lambda_{3}}{2 \lambda_{2}^{3 / 2} \sqrt{n}}+O\left(\frac{1}{n \sqrt{n}}\right)
$$


it follows from (3.6) that

$$
V_{\theta}\left(U_{\gamma, \nu}\right)=1+\frac{1}{n}\left(\frac{5 \lambda_{3}^{2}}{2 \lambda_{2}^{3}}-\frac{\lambda_{4}}{\lambda_{2}^{2}}\right)+O\left(\frac{1}{n \sqrt{n}}\right)
$$

Then it is easily seen from the first terms of (3.4), (3.7) and (3.8) that $U_{\gamma, \nu}$ is asymptotically normal with mean 0 and variance 1 .

\section{The stochastic expansion and the asymptotic variance of the MLE $\hat{\theta}_{M L}$ of $\theta$ when $\gamma$ and $\nu$ are unknown}

For given $\boldsymbol{x}=\left(x_{1}, \ldots, x_{n}\right)$ satisfying $c<\gamma<x_{(1)}$ and $x_{(n)}<\nu<d$, the likelihood function of $\theta$, $\gamma$ and $\nu$ is given by

$$
L(\theta, \gamma, \nu ; \boldsymbol{x})=\frac{1}{b^{n}(\theta, \gamma, \nu)}\left\{\prod_{i=1}^{n} a\left(x_{i}\right)\right\} \exp \left\{\theta \sum_{i=1}^{n} u\left(x_{i}\right)\right\}
$$

Let $\hat{\theta}_{M L}, \hat{\gamma}_{M L}$ and $\hat{\nu}_{M L}$ be the MLEs of $\theta, \gamma$ and $\nu$, respectively. Then it follows from (4.1) that $\hat{\gamma}_{M L}=X_{(1)}$ and $\hat{\nu}_{M L}=X_{(n)}$ and $L\left(\hat{\theta}_{M L}, X_{(1)}, X_{(n)} ; \boldsymbol{X}\right)=\sup _{\theta \in \Theta} L\left(\theta, X_{(1)}, X_{(n)} ; \boldsymbol{X}\right)$, hence $\hat{\theta}_{M L}$ satisfies the likelihood equation

$$
0=\frac{1}{n} \sum_{i=1}^{n} u\left(X_{i}\right)-\lambda_{1}\left(\hat{\theta}_{M L}, X_{(1)}, X_{(n)}\right),
$$

where $\boldsymbol{X}=\left(X_{1}, \ldots, X_{n}\right)$. Since, for $(\theta, \gamma, \nu) \in \Theta \times\left(c, X_{(1)}\right) \times\left(X_{(n)}, d\right)$

$$
\begin{aligned}
& \lambda_{1}\left(\hat{\theta}_{M L}, X_{(1)}, X_{(n)}\right) \\
& =\lambda_{1}(\theta, \gamma, \nu)+\left\{\frac{\partial}{\partial \theta} \lambda_{1}(\theta, \gamma, \nu)\right\}\left(\hat{\theta}_{M L}-\theta\right)+\left\{\frac{\partial}{\partial \gamma} \lambda_{1}(\theta, \gamma, \nu)\right\}\left(X_{(1)}-\gamma\right) \\
& +\left\{\frac{\partial}{\partial \nu} \lambda_{1}(\theta, \gamma, \nu)\right\}\left(X_{(n)}-\nu\right)+\frac{1}{2}\left[\left\{\frac{\partial^{2}}{\partial \theta^{2}} \lambda_{1}(\theta, \gamma, \nu)\right\}\left(\hat{\theta}_{M L}-\theta\right)^{2}\right. \\
& +2\left\{\frac{\partial^{2}}{\partial \theta \partial \gamma} \lambda_{1}(\theta, \gamma, \nu)\right\}\left(\hat{\theta}_{M L}-\theta\right)\left(X_{(1)}-\gamma\right)+2\left\{\frac{\partial^{2}}{\partial \theta \partial \nu} \lambda_{1}(\theta, \gamma, \nu)\right\}\left(\hat{\theta}_{M L}-\theta\right)\left(X_{(n)}-\nu\right) \\
& +\left\{\frac{\partial^{2}}{\partial \gamma^{2}} \lambda_{1}(\theta, \gamma, \nu)\right\}\left(X_{(1)}-\gamma\right)^{2}+2\left\{\frac{\partial^{2}}{\partial \gamma \partial \nu} \lambda_{1}(\theta, \gamma, \nu)\right\}\left(X_{(1)}-\gamma\right)\left(X_{(n)}-\nu\right) \\
& \left.+\left\{\frac{\partial^{2}}{\partial \nu^{2}} \lambda_{1}(\theta, \gamma, \nu)\right\}\left(X_{(n)}-\nu\right)^{2}\right]+\frac{1}{6}\left\{\frac{\partial^{3}}{\partial \theta^{3}} \lambda_{1}(\theta, \gamma)\right\}\left(\hat{\theta}_{M L}-\theta\right)^{3}+\cdots,
\end{aligned}
$$


putting $\hat{U}:=\sqrt{\lambda_{2}(\theta, \gamma, \nu) n}\left(\hat{\theta}_{M L}-\theta\right), T_{(1)}:=n\left(X_{(1)}-\gamma\right), T_{(n)}:=n\left(X_{(n)}-\nu\right)$, we have from (4.2) and (4.3)

$$
\begin{aligned}
0= & \sqrt{\frac{\lambda_{2}}{n}} Z_{1}-\sqrt{\frac{\lambda_{2}}{n}} \hat{U}-\frac{1}{n}\left(\frac{\partial \lambda_{1}}{\partial \gamma}\right) T_{(1)}-\frac{1}{n}\left(\frac{\partial \lambda_{1}}{\partial \nu}\right) T_{(n)}-\frac{\lambda_{3}}{2 \lambda_{2} n} \hat{U}^{2} \\
& -\frac{1}{\sqrt{\lambda_{2} n} n}\left(\frac{\partial \lambda_{2}}{\partial \gamma}\right) \hat{U} T_{(1)}-\frac{1}{\sqrt{\lambda_{2} n} n}\left(\frac{\partial \lambda_{2}}{\partial \nu}\right) \hat{U} T_{(n)}-\frac{\lambda_{4}}{6 \lambda_{2}^{3 / 2} n \sqrt{n}} \hat{U}^{3}+O_{p}\left(\frac{1}{n^{2}}\right),
\end{aligned}
$$

hence the stochastic expansion of $\hat{U}$ is given by

$$
\begin{aligned}
\hat{U}=Z_{1} & -\frac{1}{\sqrt{\lambda_{2} n}}\left(\frac{\partial \lambda_{1}}{\partial \gamma}\right) T_{(1)}-\frac{1}{\sqrt{\lambda_{2} n}}\left(\frac{\partial \lambda_{1}}{\partial \nu}\right) T_{(n)}-\frac{\lambda_{3}}{2 \lambda_{2}^{3 / 2} \sqrt{n}} \hat{U}^{2}-\frac{1}{\lambda_{2} n}\left(\frac{\partial \lambda_{2}}{\partial \gamma}\right) \hat{U} T_{(1)} \\
& -\frac{1}{\lambda_{2} n}\left(\frac{\partial \lambda_{2}}{\partial \nu}\right) \hat{U} T_{(n)}-\frac{\lambda_{4}}{6 \lambda_{2}^{2} n} \hat{U}^{3}+O_{p}\left(\frac{1}{n \sqrt{n}}\right) \\
=Z_{1} & -\frac{1}{\sqrt{\lambda_{2} n}}\left(\frac{\partial \lambda_{1}}{\partial \gamma}\right) T_{(1)}-\frac{1}{\sqrt{\lambda_{2} n}}\left(\frac{\partial \lambda_{1}}{\partial \nu}\right) T_{(n)} \\
& -\frac{\lambda_{3}}{2 \lambda_{2}^{3 / 2} \sqrt{n}}\left\{Z_{1}-\frac{1}{\sqrt{\lambda_{2} n}}\left(\frac{\partial \lambda_{1}}{\partial \gamma}\right) T_{(1)}-\frac{1}{\sqrt{\lambda_{2} n}}\left(\frac{\partial \lambda_{1}}{\partial \nu}\right) T_{(n)}\right\} \\
& -\frac{1}{\lambda_{2} n}\left(\frac{\partial \lambda_{2}}{\partial \gamma}\right) Z_{1} T_{(1)}-\frac{1}{\lambda_{2} n}\left(\frac{\partial \lambda_{2}}{\partial \nu}\right) Z_{1} T_{(n)}+\frac{1}{2 n}\left(\frac{\lambda_{3}^{2}}{\lambda_{2}^{3}}-\frac{\lambda_{4}}{3 \lambda_{2}^{2}}\right) Z_{1}^{3}+O_{p}\left(\frac{1}{n \sqrt{n}}\right) \\
= & Z_{1}-\frac{\lambda_{3}}{2 \lambda_{2}^{3 / 2} \sqrt{n}} Z_{1}^{2}-\frac{1}{\sqrt{\lambda_{2} n}}\left\{\left(\frac{\partial \lambda_{1}}{\partial \gamma}\right) T_{(1)}+\left(\frac{\partial \lambda_{1}}{\partial \nu}\right) T_{(n)}\right\}+\frac{1}{\lambda_{2} n} Z_{1}\left\{\delta_{1} T_{(1)}+\delta_{2} T_{(n)}\right\} \\
& +\frac{1}{2 n}\left(\frac{\lambda_{3}^{2}}{\lambda_{2}^{3}}-\frac{\lambda_{4}}{3 \lambda_{2}^{2}}\right) Z_{1}^{3}+O_{p}\left(\frac{1}{n \sqrt{n}}\right),
\end{aligned}
$$

where

$$
\delta_{1}:=\frac{\lambda_{3}}{\lambda_{2}}\left(\frac{\partial \lambda_{1}}{\partial \gamma}\right)-\frac{\partial \lambda_{2}}{\partial \gamma}, \quad \delta_{2}:=\frac{\lambda_{3}}{\lambda_{2}}\left(\frac{\partial \lambda_{1}}{\partial \nu}\right)-\frac{\partial \lambda_{2}}{\partial \nu}
$$

Since $E_{\theta, \gamma, \nu}\left(Z_{1}\right)=0, E_{\theta, \gamma, \nu}\left(Z_{1}^{2}\right)=1$ and $E_{\theta, \gamma, \nu}\left(Z_{1}^{3}\right)=\lambda_{3} /\left(\lambda_{2}^{3 / 2} \sqrt{n}\right)$ by $(3.5)$, it follows from (4.4) that

$$
\begin{aligned}
E_{\theta, \gamma, \nu}(\hat{U})=- & \frac{\lambda_{3}}{2 \lambda_{2}^{3 / 2} \sqrt{n}}-\frac{1}{\sqrt{\lambda_{2} n}}\left\{\left(\frac{\partial \lambda_{1}}{\partial \gamma}\right) E_{\theta, \gamma, \nu}\left(T_{(1)}\right)+\left(\frac{\partial \lambda_{1}}{\partial \nu}\right) E_{\theta, \gamma, \nu}\left(T_{(n)}\right)\right\} \\
& +\frac{1}{\lambda_{2} n}\left\{\delta_{1} E_{\theta, \gamma, \nu}\left(Z_{1} T_{(1)}\right)+\delta_{2} E_{\theta, \gamma, \nu}\left(Z_{1} T_{(n)}\right)\right\}+O\left(\frac{1}{n \sqrt{n}}\right) .
\end{aligned}
$$


Here, it is seen in a similar way to Akahira (2013) that

$$
\begin{aligned}
& E_{\theta, \gamma, \nu}\left(T_{(1)}\right)=\frac{1}{k(\theta, \gamma, \nu)}+\frac{A_{1}(\theta, \gamma, \nu)}{n}+O\left(\frac{1}{n^{2}}\right), \\
& E_{\theta, \gamma, \nu}\left(T_{(1)}^{2}\right)=\frac{2}{k^{2}(\theta, \gamma, \nu)}+O\left(\frac{1}{n}\right),
\end{aligned}
$$

where

$$
A_{1}(\theta, \gamma, \nu):=-\frac{1}{k^{2}(\theta, \gamma, \nu)}\left\{\frac{c_{\theta}(\gamma)}{a(\gamma)}+k(\theta, \gamma, \nu)\right\}
$$

with $k(\theta, \gamma, \nu)=a(\gamma) e^{\theta u(\gamma)} / b(\theta, \gamma, \nu)$ and $c_{\theta}(\gamma)=a^{\prime}(\gamma)+\theta a(\gamma) u^{\prime}(\gamma)$. Since the second order asymptotic cumulative distribution function of $T_{(n)}$ is given by

$$
\begin{aligned}
F_{T_{(n)}}(t) & =P_{\theta, \gamma, \nu}\left\{T_{(n)} \leq t\right\}=P_{\theta, \gamma, \nu}\left\{n\left(X_{(n)}-\nu\right) \leq t\right\} \\
& =\left\{1-\int_{\nu+\frac{t}{n}}^{\nu} \frac{1}{b(\theta, \gamma, \nu)} a(x) e^{\theta u(x)} d x\right\}^{n} \\
& =\left[\exp \left\{\frac{a(\nu) e^{\theta u(\nu)}}{b(\theta, \gamma, \nu)} t\right\}\right] \cdot\left[1-\frac{e^{\theta u(\nu)} t^{2}}{2 b^{2}(\theta, \gamma, \nu) n}\left\{c_{\theta}(\nu) b(\theta, \gamma, \nu)+a^{2}(\nu) e^{\theta u(\nu)}\right\}+O\left(\frac{1}{n^{2}}\right)\right]
\end{aligned}
$$

for $t<0$, where $c_{\theta}(\nu)=a^{\prime}(\nu)+\theta a(\nu) u^{\prime}(\nu)$, it follows that the second order asymptotic density of $T_{(n)}$ is

$$
\begin{array}{r}
f_{T_{(n)}}(t)=\tilde{k}(\theta, \gamma, \nu) e^{\tilde{k}(\theta, \gamma, \nu) t}+\frac{\tilde{k}(\theta, \gamma, \nu)}{a(\nu) b(\theta, \gamma, \nu) n}\left\{c_{\theta}(\nu) b(\theta, \gamma, \nu)-a^{2}(\nu) e^{\theta u(\nu)}\right\} \\
\cdot\left\{t+\frac{\tilde{k}(\theta, \gamma, \nu)}{2} t^{2}\right\} e^{\tilde{k}(\theta, \gamma, \nu) t}+O\left(\frac{1}{n^{2}}\right)
\end{array}
$$

for $t<0$, where $\tilde{k}(\theta, \gamma, \nu):=a(\nu) e^{\theta u(\nu)} / b(\theta, \gamma, \nu)$. Then

$$
\begin{aligned}
& E_{\theta, \gamma, \nu}\left(T_{(n)}\right)=-\frac{1}{\tilde{k}(\theta, \gamma, \nu)}-\frac{A_{2}(\theta, \gamma, \nu)}{n}+O\left(\frac{1}{n^{2}}\right), \\
& E_{\theta, \gamma, \nu}\left(T_{(n)}^{2}\right)=\frac{2}{\tilde{k}^{2}(\theta, \gamma, \nu)}+O\left(\frac{1}{n}\right)
\end{aligned}
$$

where

$$
A_{2}(\theta, \gamma, \nu):=\frac{1}{\tilde{k}^{2}(\theta, \gamma, \nu)}\left\{\frac{c_{\theta}(\nu)}{a(\nu)}-\tilde{k}(\theta, \gamma, \nu)\right\} .
$$


Let $Y_{1}, \ldots, Y_{n-1}$ be a random permutation of the $(n-1)$ ! permutations of $X_{(1)}, \ldots, X_{(n-1)}$ such that conditional on $X_{(n)}=x_{(n)}$, the $Y_{1}, \ldots, Y_{n-1}$ are i.i.d. random variables with a density

$$
g\left(y ; \theta, \gamma, x_{(n)}\right)=\frac{a(y) e^{\theta u(y)}}{b\left(\theta, \gamma, x_{(n)}\right)} \quad \text { for } c<\gamma<y<x_{(n)}<\nu<d,
$$

(see Quesenberry (1975) and Bar-Lev (1984)). Then the conditional expectation of $Z_{1}$, given $T_{(n)}$ is obtained by

$$
\begin{aligned}
E_{\theta, \gamma, \nu}\left(Z_{1} \mid T_{(n)}\right) & =\frac{1}{\sqrt{\lambda_{2} n}} \sum_{i=1}^{n}\left\{E_{\theta, \gamma, \nu}\left[u\left(X_{i}\right) \mid T_{(n)}\right]-\lambda_{1}\right\} \\
& =\frac{1}{\sqrt{\lambda_{2} n}} \sum_{i=1}^{n}\left\{E_{\theta, \gamma, \nu}\left[u\left(X_{(i)}\right) \mid T_{(n)}\right]-\lambda_{1}\right\} \\
& =\frac{1}{\sqrt{\lambda_{2} n}}\left\{u\left(X_{(n)}\right)+\sum_{i=1}^{n-1} E_{\theta, \gamma, \nu}\left[u\left(Y_{i}\right) \mid T_{(n)}\right]-n \lambda_{1}\right\}
\end{aligned}
$$

where $\lambda_{i}=\lambda_{i}(\theta, \gamma, \nu)(i=1,2)$. Since, for $i=1, \ldots, n-1$,

$$
\begin{aligned}
E_{\theta, \gamma, \nu}\left[u\left(Y_{i}\right) \mid T_{(n)}\right] & =\int_{\gamma}^{X_{(n)}} u(y) \frac{a(y) e^{\theta u(y)}}{b\left(\theta, \gamma, X_{(n)}\right)} d y \\
& =\frac{\partial}{\partial \theta} \log b\left(\theta, \gamma, X_{(n)}\right)=\lambda_{1}\left(\theta, \gamma, X_{(n)}\right)=:{\hat{\lambda_{1}}}^{(n)} \quad \text { (say), }
\end{aligned}
$$

it follows from (4.11) that

$$
E_{\theta, \gamma, \nu}\left(Z_{1} \mid T_{(n)}\right)=\frac{1}{\sqrt{\lambda_{2} n}}\left\{u\left(X_{(n)}\right)+(n-1){\hat{\lambda_{1}}}^{(n)}\right\}-\frac{\lambda_{1} \sqrt{n}}{\sqrt{\lambda_{2}}}
$$

hence, from (4.8) and (4.11)

$$
\begin{aligned}
E_{\theta, \gamma, \nu}\left(Z_{1} T_{(n)}\right)= & E_{\theta, \gamma, \nu}\left[T_{(n)} E_{\theta, \gamma, \nu}\left(Z_{1} \mid T_{(n)}\right)\right] \\
= & \frac{1}{\sqrt{\lambda_{2} n}}\left\{E_{\theta, \gamma, \nu}\left[u\left(X_{(n)}\right) T_{(n)}\right]+(n-1) E_{\theta, \gamma, \nu}\left({\hat{\lambda_{1}}}^{(n)} T_{(n)}\right)\right\} \\
& \quad-\sqrt{\frac{n}{\lambda_{2}}} \lambda_{1}\left\{-\frac{1}{\tilde{k}}-\frac{A_{2}}{n}+O\left(\frac{1}{n^{2}}\right)\right\},
\end{aligned}
$$


where $\tilde{k}=\tilde{k}(\theta, \gamma, \nu)$. Since, by the Taylor expansion

$$
\begin{aligned}
u\left(X_{(n)}\right) & =u(\nu)+\frac{u^{\prime}(\nu)}{n} T_{(n)}+\frac{u^{\prime \prime}(\nu)}{2 n^{2}} T_{(n)}^{2}+O_{p}\left(\frac{1}{n^{3}}\right), \\
{\hat{\lambda_{1}}}^{(n)} & =\lambda_{1}\left(\theta, \gamma, X_{(n)}\right) \\
& =\lambda_{1}(\theta, \gamma, \nu)+\frac{1}{n}\left\{\frac{\partial}{\partial \nu} \lambda_{1}(\theta, \gamma, \nu)\right\} T_{(n)}+\frac{1}{2 n^{2}}\left\{\frac{\partial^{2}}{\partial \nu^{2}} \lambda_{1}(\theta, \gamma, \nu)\right\} T_{(n)}^{2}+O_{p}\left(\frac{1}{n^{3}}\right),
\end{aligned}
$$

it follows from (4.8) and (4.9) that

$$
\begin{aligned}
E_{\theta, \gamma, \nu}\left[u\left(X_{(n)}\right) T_{(n)}\right] & =u(\nu) E_{\theta, \gamma, \nu}\left(T_{(n)}\right)+\frac{u^{\prime}(\nu)}{n} E_{\theta, \gamma, \nu}\left(T_{(n)}^{2}\right)+O\left(\frac{1}{n^{2}}\right) \\
& =-\frac{u(\nu)}{\tilde{k}}-\left\{A_{2} u(\nu)-\frac{2 u^{\prime}(\nu)}{\tilde{k}^{2}}\right\} \frac{1}{n}+O\left(\frac{1}{n^{2}}\right), \\
E_{\theta, \gamma, \nu}\left({\hat{\lambda_{1}}}^{(n)} T_{(n)}\right) & =-\frac{\lambda_{1}}{\tilde{k}}-\left\{\lambda_{1} A_{2}-\frac{2}{\tilde{k}^{2}}\left(\frac{\partial \lambda_{1}}{\partial \nu}\right)\right\} \frac{1}{n}+O\left(\frac{1}{n^{2}}\right),
\end{aligned}
$$

where $\tilde{k}=\tilde{k}(\theta, \gamma, \nu), A_{2}=A_{2}(\theta, \gamma, \nu)$ and $\lambda_{1}=\lambda_{1}(\theta, \gamma, \nu)$. From (4.12) to (4.14) we obtain

$$
E_{\theta, \gamma, \nu}\left(Z_{1} T_{(n)}\right)=-\frac{1}{\tilde{k} \sqrt{\lambda_{2} n}}\left\{u(\nu)-\lambda_{1}-\frac{2}{\tilde{k}}\left(\frac{\partial \lambda_{1}}{\partial \nu}\right)\right\}+O\left(\frac{1}{n \sqrt{n}}\right) .
$$

On the other hand, it is shown in a similar way to Akahira (2013) that

$$
E_{\theta, \gamma, \nu}\left(Z_{1} T_{(1)}\right)=\frac{1}{k \sqrt{\lambda_{2} n}}\left\{u(\gamma)-\lambda_{1}+\frac{2}{k}\left(\frac{\partial \lambda_{1}}{\partial \gamma}\right)\right\}+O\left(\frac{1}{n \sqrt{n}}\right)
$$

where $k=k(\theta, \gamma, \nu)$. From (4.5), (4.6), (4.8), (4.15) and (4.16) we obtain

$$
E_{\theta, \gamma, \nu}(\hat{U})=-\frac{1}{\sqrt{\lambda_{2} n}}\left\{\frac{1}{k}\left(\frac{\partial \lambda_{1}}{\partial \gamma}\right)-\frac{1}{\tilde{k}}\left(\frac{\partial \lambda_{1}}{\partial \nu}\right)+\frac{\lambda_{3}}{2 \lambda_{2}}\right\}+O\left(\frac{1}{n \sqrt{n}}\right) .
$$

Since, by (4.4)

$$
\begin{aligned}
\hat{U}=Z_{1} & -\frac{\lambda_{3}}{2 \lambda_{2}^{3 / 2} \sqrt{n}} Z_{1}^{2}-\frac{1}{\sqrt{\lambda_{2} n}}\left\{\left(\frac{\partial \lambda_{1}}{\partial \gamma}\right) T_{(1)}+\left(\frac{\partial \lambda_{1}}{\partial \nu}\right) T_{(n)}\right\} \\
& +\frac{\delta_{1}}{\lambda_{2} n} Z_{1} T_{(1)}+\frac{\delta_{2}}{\lambda_{2} n} Z_{1} T_{(n)}+\frac{1}{2 n}\left(\frac{\lambda_{3}^{2}}{\lambda_{2}^{3}}-\frac{\lambda_{4}}{3 \lambda_{2}^{2}}\right) Z_{1}^{3}+O_{p}\left(\frac{1}{n \sqrt{n}}\right)
\end{aligned}
$$


it follows that

$$
\begin{aligned}
& E_{\theta, \gamma, \nu}\left(\hat{U}^{2}\right) \\
& =E_{\theta, \gamma, \nu}\left(Z_{1}^{2}\right)-\frac{1}{\sqrt{\lambda_{2} n}}\left\{2\left(\frac{\partial \lambda_{1}}{\partial \gamma}\right) E_{\theta, \gamma, \nu}\left(Z_{1} T_{(1)}\right)+2\left(\frac{\partial \lambda_{1}}{\partial \nu}\right) E_{\theta, \gamma, \nu}\left(Z_{1} T_{(n)}\right)+\frac{\lambda_{3}}{\lambda_{2}} E_{\theta, \gamma, \nu}\left(Z_{1}^{3}\right)\right\} \\
& +\frac{1}{\lambda_{2} n}\left\{\left(\frac{\partial \lambda_{1}}{\partial \gamma}\right)^{2} E_{\theta, \gamma, \nu}\left(T_{(1)}^{2}\right)+2\left(\frac{\partial \lambda_{1}}{\partial \gamma}\right)\left(\frac{\partial \lambda_{1}}{\partial \nu}\right) E_{\theta, \gamma, \nu}\left(T_{(1)} T_{(n)}\right)+\left(\frac{\partial \lambda_{1}}{\partial \nu}\right)^{2} E_{\theta, \gamma, \nu}\left(T_{(n)}^{2}\right)\right\} \\
& +\frac{1}{\lambda_{2} n}\left\{\frac{\lambda_{3}}{\lambda_{2}}\left(\frac{\partial \lambda_{1}}{\partial \gamma}\right)+2 \delta_{1}\right\} E_{\theta, \gamma, \nu}\left(Z_{1}^{2} T_{(1)}\right)+\frac{1}{\lambda_{2} n}\left\{\frac{\lambda_{3}}{\lambda_{2}}\left(\frac{\partial \lambda_{1}}{\partial \nu}\right)+2 \delta_{2}\right\} E_{\theta, \gamma, \nu}\left(Z_{1}^{2} T_{(n)}\right) \\
& +\frac{\lambda_{3}^{2}}{4 \lambda_{2}^{3} n} E_{\theta, \gamma, \nu}\left(Z_{1}^{4}\right)+\frac{1}{n}\left(\frac{\lambda_{3}^{2}}{\lambda_{2}^{3}}-\frac{\lambda_{4}}{3 \lambda_{2}^{2}}\right) E_{\theta, \gamma, \nu}\left(Z_{1}^{4}\right)+O\left(\frac{1}{n \sqrt{n}}\right) .
\end{aligned}
$$

Since $T_{(1)}$ and $T_{(n)}$ are asymptotically independent, it follows that

$$
\begin{aligned}
E_{\theta, \gamma, \nu}\left(T_{(1)} T_{(n)}\right) & =E_{\theta, \gamma, \nu}\left(T_{(1)}\right) E_{\theta, \gamma, \nu}\left(T_{(n)}\right)+O\left(\frac{1}{n}\right) \\
& =-\frac{1}{k \tilde{k}}+O\left(\frac{1}{n}\right) .
\end{aligned}
$$

Here

$$
\begin{aligned}
E_{\theta, \gamma, \nu}\left(Z_{1}^{2} \mid T_{(n)}\right)= & E_{\theta, \gamma, \nu}\left[\frac{1}{\lambda_{2} n}\left\{\sum_{i=1}^{n}\left(u\left(X_{i}\right)-\lambda_{1}\right)\right\}^{2} \mid T_{(n)}\right] \\
= & \frac{1}{\lambda_{2} n} E_{\theta, \gamma, \nu}\left[\left\{\sum_{i=1}^{n-1}\left(u\left(Y_{i}\right)-\lambda_{1}\right)+u\left(X_{(n)}\right)-\lambda_{1}\right\}^{2} \mid T_{(n)}\right] \\
= & \frac{1}{\lambda_{2} n}\left\{u\left(X_{(n)}\right)-\lambda_{1}\right\}^{2}+\frac{2}{\lambda_{2} n}\left\{u\left(X_{(n)}\right)-\lambda_{1}\right\} \sum_{i=1}^{n-1} E_{\theta, \gamma, \nu}\left[u\left(Y_{i}\right)-\lambda_{1} \mid T_{(n)}\right] \\
& +\frac{1}{\lambda_{2} n} \sum_{i=1}^{n-1} E_{\theta, \gamma, \nu}\left[\left\{u\left(Y_{i}\right)-\lambda_{1}\right\}^{2} \mid T_{(n)}\right] \\
& +\frac{1}{\lambda_{2} n} \sum_{i \neq j} \sum_{1 \leq i, j \leq n-1} E_{\theta, \gamma, \nu}\left[\left\{u\left(Y_{i}\right)-\lambda_{1}\right\}\left\{u\left(Y_{j}\right)-\lambda_{1}\right\} \mid T_{(n)}\right]
\end{aligned}
$$

For $1 \leq i \leq n-1$, we have

$$
\begin{aligned}
E_{\theta, \gamma, \nu}\left[u\left(Y_{i}\right)-\lambda_{1} \mid T_{(n)}\right] & =E_{\theta, \gamma, \nu}\left[u\left(Y_{i}\right) \mid T_{(n)}\right]-\lambda_{1}=\lambda_{1}\left(\theta, \gamma, X_{(n)}\right)-\lambda_{1}(\theta, \gamma, \nu) \\
& =\hat{\lambda}^{(n)}-\lambda_{1}=\left(\frac{\partial \lambda_{1}}{\partial \nu}\right) \frac{T_{(n)}}{n}+O_{p}\left(\frac{1}{n^{2}}\right)
\end{aligned}
$$


and, for $i \neq j$ and $1 \leq i, j \leq n-1$

$$
\begin{aligned}
& E_{\theta, \gamma, \nu}\left[\left\{u\left(Y_{i}\right)-\lambda_{1}\right\}\left\{u\left(Y_{j}\right)-\lambda_{1}\right\} \mid T_{(n)}\right] \\
= & E_{\theta, \gamma, \nu}\left[u\left(Y_{i}\right)-\lambda_{1} \mid T_{(n)}\right] E_{\theta, \gamma, \nu}\left[u\left(Y_{j}\right)-\lambda_{1} \mid T_{(n)}\right] \\
= & \left(\frac{\partial \lambda_{1}}{\partial \nu}\right)^{2} \frac{T_{(n)}^{2}}{n^{2}}+O_{p}\left(\frac{1}{n^{3}}\right) .
\end{aligned}
$$

Since, for $i=1, \ldots, n-1$

$$
\begin{aligned}
E_{\theta, \gamma, \nu}\left[u^{2}\left(Y_{i}\right) \mid T_{(n)}\right] & =\int_{\gamma}^{X_{(n)}} u^{2}(y) \frac{a(y) e^{\theta u(y)}}{b\left(\theta, \gamma, X_{(n)}\right)} d y \\
& =\frac{1}{b\left(\theta, \gamma, X_{(n)}\right)} \frac{\partial^{2}}{\partial \theta^{2}} b\left(\theta, \gamma, X_{(n)}\right) \\
& =\lambda_{1}^{2}\left(\theta, \gamma, X_{(n)}\right)+\lambda_{2}\left(\theta, \gamma, X_{(n)}\right) \\
& =:{\hat{\lambda_{1}}}^{(n)^{2}}+{\hat{\lambda_{2}}}^{(n)}
\end{aligned}
$$

where ${\hat{\lambda_{i}}}^{(n)}=\lambda_{i}\left(\theta, \gamma, X_{(n)}\right)(i=1,2)$, we have for $i=1, \ldots, n-1$

$$
\begin{aligned}
& E_{\theta, \gamma, \nu}\left[\left\{u\left(Y_{i}\right)-\lambda_{1}\right\}^{2} \mid T_{(n)}\right] \\
= & E_{\theta, \gamma, \nu}\left[u^{2}\left(Y_{i}\right) \mid T_{(n)}\right]-2 \lambda_{1} E_{\theta, \gamma, \nu}\left[u\left(Y_{i}\right) \mid T_{(n)}\right]+\lambda_{1}^{2} \\
= & {\hat{\lambda_{1}}}^{(n)^{2}}+{\hat{\lambda_{2}}}^{(n)}-2 \lambda_{1}{\hat{\lambda_{1}}}^{(n)}+\lambda_{1}^{2} \\
= & \left\{\lambda_{1}+\frac{1}{n}\left(\frac{\partial \lambda_{1}}{\partial \nu}\right) T_{(n)}+O_{p}\left(\frac{1}{n^{2}}\right)\right\}^{2}+\lambda_{2}+\frac{1}{n}\left(\frac{\partial \lambda_{2}}{\partial \nu}\right) T_{(n)}-2 \lambda_{1}^{2}-\frac{2}{n}\left(\frac{\partial \lambda_{1}}{\partial \nu}\right) \lambda_{1} T_{(n)} \\
& \quad+\lambda_{1}^{2}+O_{p}\left(\frac{1}{n^{2}}\right) \\
= & \lambda_{2}+\frac{1}{n}\left(\frac{\partial \lambda_{2}}{\partial \nu}\right) T_{(n)}+O_{p}\left(\frac{1}{n^{2}}\right) .
\end{aligned}
$$


From (4.20) to (4.23) we obtain

$$
\begin{aligned}
E_{\theta, \gamma, \nu}\left(Z_{1}^{2} \mid T_{(n)}\right)= & \frac{1}{\lambda_{2} n}\left\{u\left(X_{(n)}\right)-\lambda_{1}\right\}^{2}+\frac{2}{\lambda_{2}}\left\{u\left(X_{(n)}\right)-\lambda_{1}\right\}\left(1-\frac{1}{n}\right)\left\{\left(\frac{\partial \lambda_{1}}{\partial \nu}\right) \frac{T_{(n)}}{n}+O_{p}\left(\frac{1}{n^{2}}\right)\right\} \\
& +\frac{1}{\lambda_{2}}\left(1-\frac{1}{n}\right)\left\{\lambda_{2}+\frac{1}{n}\left(\frac{\partial \lambda_{2}}{\partial \nu}\right) T_{(n)}+O_{p}\left(\frac{1}{n^{2}}\right)\right\} \\
& +\frac{1}{\lambda_{2} n}\left(1-\frac{1}{n}\right)\left(1-\frac{2}{n}\right)\left\{\left(\frac{\partial \lambda_{1}}{\partial \nu}\right)^{2} T_{(n)}^{2}+O_{p}\left(\frac{1}{n}\right)\right\} \\
=1 & -\frac{1}{n}+\frac{1}{\lambda_{2} n}\left\{u\left(X_{(n)}\right)-\lambda_{1}\right\}^{2}+\frac{2}{\lambda_{2} n}\left\{u\left(X_{(n)}\right)-\lambda_{1}\right\}\left(\frac{\partial \lambda_{1}}{\partial \nu}\right) T_{(n)} \\
& +\frac{1}{\lambda_{2} n}\left(\frac{\partial \lambda_{2}}{\partial \nu}\right) T_{(n)}+\frac{1}{\lambda_{2} n}\left(\frac{\partial \lambda_{1}}{\partial \nu}\right)^{2} T_{(n)}^{2}+O_{p}\left(\frac{1}{n^{2}}\right) \\
=1 & +O_{p}\left(\frac{1}{n}\right),
\end{aligned}
$$

hence, by (4.8)

$$
E_{\theta, \gamma, \nu}\left(Z_{1}^{2} T_{(n)}\right)=E_{\theta, \gamma, \nu}\left[T_{(n)} E_{\theta, \gamma, \nu}\left(Z_{1}^{2} \mid T_{(n)}\right)\right]=E_{\theta, \gamma, \nu}\left(T_{(n)}\right)+O\left(\frac{1}{n}\right)=-\frac{1}{\tilde{k}}+O\left(\frac{1}{n}\right) .
$$

On the other hand, it is shown a similar way to Akahira (2013) that

$$
E_{\theta, \gamma, \nu}\left(Z_{1}^{2} T_{(1)}\right)=\frac{1}{k}+O\left(\frac{1}{n}\right)
$$

Substituting (3.5), (4.7), (4.9), (4.15), (4.16), (4.19), (4.24) and (4.25) for (4.18) we obtain

$$
\begin{aligned}
E_{\theta, \gamma, \nu}\left(\hat{U}^{2}\right)=1 & -\frac{1}{\sqrt{\lambda_{2} n}}\left[2\left(\frac{\partial \lambda_{1}}{\partial \gamma}\right) \frac{1}{k \sqrt{\lambda_{2} n}}\left\{u(\gamma)-\lambda_{1}+\frac{2}{k}\left(\frac{\partial \lambda_{1}}{\partial \gamma}\right)\right\}\right. \\
& \left.+2\left(\frac{\partial \lambda_{1}}{\partial \nu}\right)\left\{-\frac{1}{\tilde{k} \sqrt{\lambda_{2} n}}\left(u(\nu)-\lambda_{1}-\frac{2}{\tilde{k}}\left(\frac{\partial \lambda_{1}}{\partial \nu}\right)\right)\right\}+\frac{\lambda_{3}^{2}}{\lambda_{2}^{5 / 2} \sqrt{n}}\right] \\
& +\frac{2}{\lambda_{2} n}\left\{\frac{1}{k^{2}}\left(\frac{\partial \lambda_{1}}{\partial \gamma}\right)^{2}-\left(\frac{1}{k \tilde{k}}\right)\left(\frac{\partial \lambda_{1}}{\partial \gamma}\right)\left(\frac{\partial \lambda_{1}}{\partial \nu}\right)+\frac{1}{\tilde{k}^{2}}\left(\frac{\partial \lambda_{1}}{\partial \nu}\right)^{2}\right\} \\
& +\frac{1}{k \lambda_{2} n}\left\{\frac{\lambda_{3}}{\lambda_{2}}\left(\frac{\partial \lambda_{1}}{\partial \gamma}\right)+2 \delta_{1}\right\}-\frac{1}{\tilde{k} \lambda_{2} n}\left\{\frac{\lambda_{3}}{\lambda_{2}}\left(\frac{\partial \lambda_{1}}{\partial \nu}\right)+2 \delta_{2}\right\} \\
& +\frac{15 \lambda_{3}^{2}}{4 \lambda_{2}^{3} n}-\frac{\lambda_{4}}{\lambda_{2}^{2} n}+O\left(\frac{1}{n^{2}}\right) \\
=1 & \frac{2}{\lambda_{2} n}\left[\frac{1}{k}\left(\frac{\partial \lambda_{1}}{\partial \gamma}\right)\left\{u(\gamma)-\lambda_{1}+\frac{1}{k}\left(\frac{\partial \lambda_{1}}{\partial \gamma}\right)\right\}-\frac{1}{\tilde{k}}\left(\frac{\partial \lambda_{1}}{\partial \nu}\right)\left\{u(\nu)-\lambda_{1}-\frac{1}{\tilde{k}}\left(\frac{\partial \lambda_{1}}{\partial \nu}\right)\right\}\right]
\end{aligned}
$$




$$
\begin{aligned}
& -\frac{2}{k \tilde{k} \lambda_{2} n}\left(\frac{\partial \lambda_{1}}{\partial \gamma}\right)\left(\frac{\partial \lambda_{1}}{\partial \nu}\right)+\frac{3 \lambda_{3}}{\lambda_{2}^{2} n}\left\{\frac{1}{k}\left(\frac{\partial \lambda_{1}}{\partial \gamma}\right)-\frac{1}{\tilde{k}}\left(\frac{\partial \lambda_{1}}{\partial \nu}\right)\right\} \\
& -\frac{2}{\lambda_{2} n}\left\{\frac{1}{k}\left(\frac{\partial \lambda_{2}}{\partial \gamma}\right)-\frac{1}{\tilde{k}}\left(\frac{\partial \lambda_{2}}{\partial \nu}\right)\right\}+\frac{11 \lambda_{3}^{2}}{4 \lambda_{2}^{3} n}-\frac{\lambda_{4}}{\lambda_{2}^{2} n}+O\left(\frac{1}{n^{2}}\right) .
\end{aligned}
$$

In order to adjust $\hat{\theta}_{M L}$ such that $\hat{\theta}_{M L}$ has the same asymptotic bias as that of $\hat{\theta}_{M L}^{\gamma, \nu}$ given by (3.7), we define

$$
\begin{array}{r}
\hat{\theta}_{M L}^{*}=\hat{\theta}_{M L}+\frac{1}{\lambda_{2}\left(\hat{\theta}_{M L}, X_{(1)}, X_{(n)}\right) n}\left\{\frac{1}{k\left(\hat{\theta}_{M L}, X_{(1)}, X_{(n)}\right)} \frac{\partial \lambda_{1}}{\partial \gamma}\left(\hat{\theta}_{M L}, X_{(1)}, X_{(n)}\right)\right. \\
\left.-\frac{1}{\tilde{k}\left(\hat{\theta}_{M L}, X_{(1)}, X_{(n)}\right)} \frac{\partial \lambda_{1}}{\partial \nu}\left(\hat{\theta}_{M L}, X_{(1)}, X_{(n)}\right)\right\} .
\end{array}
$$

Put $\hat{\lambda_{2}}=\hat{\lambda_{2}}\left(\hat{\theta}_{M L}, X_{(1)}, X_{(n)}\right), \hat{k}=k\left(\hat{\theta}_{M L}, X_{(1)}, X_{(n)}\right)$ and $\hat{\tilde{k}}=\tilde{k}\left(\hat{\theta}_{M L}, X_{(1)}, X_{(n)}\right)$. Letting

$$
\frac{\partial \hat{\lambda_{1}}}{\partial \gamma}=\frac{\partial \lambda_{1}}{\partial \gamma}\left(\hat{\theta}_{M L}, X_{(1)}, X_{(n)}\right), \quad \frac{\partial \hat{\lambda_{1}}}{\partial \nu}=\frac{\partial \lambda_{1}}{\partial \nu}\left(\hat{\theta}_{M L}, X_{(1)}, X_{(n)}\right)
$$

we have

$$
\begin{aligned}
& \frac{\sqrt{\lambda_{2}}}{\hat{\lambda_{2}}}\left(\frac{1}{\hat{k}} \frac{\partial \hat{\lambda_{1}}}{\partial \gamma}-\frac{1}{\hat{\tilde{k}}} \frac{\partial \hat{\lambda_{1}}}{\partial \nu}\right) \\
& =\frac{1}{\sqrt{\lambda_{2}}}\left\{\frac{1}{k}\left(\frac{\partial \lambda_{1}}{\partial \gamma}\right)-\frac{1}{\tilde{k}}\left(\frac{\partial \lambda_{1}}{\partial \nu}\right)+\frac{1}{\sqrt{\lambda_{2} n}}\left(\frac{1}{k}\left(\frac{\partial^{2} \lambda_{1}}{\partial \gamma \partial \theta}\right)-\frac{1}{\hat{k}}\left(\frac{\partial^{2} \lambda_{1}}{\partial \nu \partial \theta}\right)\right) \hat{U}\right. \\
& \quad-\frac{1}{\sqrt{\lambda_{2} n}}\left(\frac{1}{k^{2}}\left(\frac{\partial k}{\partial \theta}\right)\left(\frac{\partial \lambda_{1}}{\partial \gamma}\right)-\frac{1}{\tilde{k}^{2}}\left(\frac{\partial \tilde{k}}{\partial \theta}\right)\left(\frac{\partial \lambda_{1}}{\partial \nu}\right)\right) \hat{U} \\
& \left.\quad-\frac{\lambda_{3}}{\lambda_{2}^{3 / 2} \sqrt{n}}\left(\frac{1}{k}\left(\frac{\partial \lambda_{1}}{\partial \gamma}\right)-\frac{1}{\tilde{k}}\left(\frac{\partial \lambda_{1}}{\partial \nu}\right)\right) \hat{U}+O_{p}\left(\frac{1}{n}\right)\right\}
\end{aligned}
$$

From (4.27) and (4.28) we obtain the stochastic expansion

$$
\begin{aligned}
\hat{U}^{*} & :=\sqrt{\lambda_{2} n}\left(\hat{\theta}_{M L}^{*}-\theta\right)=\sqrt{\lambda_{2} n}\left(\hat{\theta}_{M L}-\theta\right)+\frac{\sqrt{\lambda_{2}}}{\hat{\lambda_{2} \sqrt{n}}}\left\{\frac{1}{\hat{k}}\left(\frac{\partial \hat{\lambda_{1}}}{\partial \gamma}\right)-\frac{1}{\hat{\tilde{k}}}\left(\frac{\partial \hat{\lambda_{1}}}{\partial \nu}\right)\right\} \\
& =\hat{U}+\frac{1}{\sqrt{\lambda_{2} n}}\left\{\frac{1}{k}\left(\frac{\partial \lambda_{1}}{\partial \gamma}\right)-\frac{1}{\tilde{k}}\left(\frac{\partial \lambda_{1}}{\partial \nu}\right)\right\} \\
& -\frac{1}{\lambda_{2} n}\left\{\frac{\delta_{1}}{k}-\frac{\delta_{2}}{\tilde{k}}+\frac{1}{k^{2}}\left(\frac{\partial k}{\partial \theta}\right)\left(\frac{\partial \lambda_{1}}{\partial \gamma}\right)-\frac{1}{\tilde{k}^{2}}\left(\frac{\partial \tilde{k}}{\partial \theta}\right)\left(\frac{\partial \lambda_{1}}{\partial \nu}\right)\right\} Z_{1}+O_{p}\left(\frac{1}{n \sqrt{n}}\right),
\end{aligned}
$$


where $\hat{U}$ is given by (4.4). From (4.17) we have

$$
\begin{aligned}
E_{\theta, \gamma, \nu}\left(\hat{U}^{*}\right)= & E_{\theta, \gamma, \nu}(\hat{U})+\frac{1}{\sqrt{\lambda_{2} n}}\left\{\frac{1}{k}\left(\frac{\partial \lambda_{1}}{\partial \gamma}\right)-\frac{1}{\tilde{k}}\left(\frac{\partial \lambda_{1}}{\partial \nu}\right)\right\} \\
& \quad-\frac{1}{\lambda_{2} n}\left\{\frac{\delta_{1}}{k}-\frac{\delta_{2}}{\tilde{k}}+\frac{1}{k^{2}}\left(\frac{\partial k}{\partial \theta}\right)\left(\frac{\partial \lambda_{1}}{\partial \gamma}\right)-\frac{1}{\tilde{k}^{2}}\left(\frac{\partial \tilde{k}}{\partial \theta}\right)\left(\frac{\partial \lambda_{1}}{\partial \nu}\right)\right\} E_{\theta, \gamma, \nu}\left(Z_{1}\right)+O\left(\frac{1}{n \sqrt{n}}\right) \\
= & -\frac{\lambda_{3}}{2 \lambda_{2}^{3 / 2} \sqrt{n}}+O\left(\frac{1}{n \sqrt{n}}\right),
\end{aligned}
$$

which is seen to be the same asymptotic bias as that of $U_{\gamma, \nu}$ given by (3.7). Then it follows from (4.17), (4.26) and (4.29) that

$$
\begin{aligned}
& E_{\theta, \gamma, \nu}\left(\hat{U}^{*^{2}}\right) \\
& =1-\frac{2}{\lambda_{2} n}\left[\frac{1}{k}\left(\frac{\partial \lambda_{1}}{\partial \gamma}\right)\left\{u(\gamma)-\lambda_{1}+\frac{1}{k}\left(\frac{\partial \lambda_{1}}{\partial \gamma}\right)\right\}-\frac{1}{\tilde{k}}\left(\frac{\partial \lambda_{1}}{\partial \nu}\right)\left\{u(\nu)-\lambda_{1}-\frac{1}{\tilde{k}}\left(\frac{\partial \lambda_{1}}{\partial \nu}\right)\right\}\right] \\
& -\frac{2}{k \tilde{k} \lambda_{2} n}\left(\frac{\partial \lambda_{1}}{\partial \gamma}\right)\left(\frac{\partial \lambda_{1}}{\partial \nu}\right)+\frac{3 \lambda_{3}}{\lambda_{2}^{2} n}\left\{\frac{1}{k}\left(\frac{\partial \lambda_{1}}{\partial \gamma}\right)-\frac{1}{\tilde{k}}\left(\frac{\partial \lambda_{1}}{\partial \nu}\right)\right\} \\
& -\frac{2}{\lambda_{2} n}\left\{\frac{1}{k}\left(\frac{\partial \lambda_{2}}{\partial \gamma}\right)-\frac{1}{\tilde{k}}\left(\frac{\partial \lambda_{2}}{\partial \nu}\right)\right\}+\frac{11 \lambda_{3}^{2}}{4 \lambda_{2}^{3} n}-\frac{\lambda_{4}}{\lambda_{2}^{2} n} \\
& -\frac{2}{\lambda_{2} n}\left\{\frac{\delta_{1}}{k}-\frac{\delta_{2}}{\tilde{k}}+\frac{1}{k^{2}}\left(\frac{\partial k}{\partial \theta}\right)\left(\frac{\partial \lambda_{1}}{\partial \gamma}\right)-\frac{1}{\tilde{k}^{2}}\left(\frac{\partial \tilde{k}}{\partial \theta}\right)\left(\frac{\partial \lambda_{1}}{\partial \nu}\right)\right\} \\
& +\frac{1}{\lambda_{2} n}\left\{\frac{1}{k}\left(\frac{\partial \lambda_{1}}{\partial \gamma}\right)-\frac{1}{\tilde{k}}\left(\frac{\partial \lambda_{1}}{\partial \nu}\right)\right\}^{2} \\
& +\frac{2}{\sqrt{\lambda_{2} n}}\left\{\frac{1}{k}\left(\frac{\partial \lambda_{1}}{\partial \gamma}\right)-\frac{1}{\tilde{k}}\left(\frac{\partial \lambda_{1}}{\partial \nu}\right)\right\} \\
& \cdot\left\{-\frac{1}{\sqrt{\lambda_{2} n}}\left(\frac{1}{k}\left(\frac{\partial \lambda_{1}}{\partial \gamma}\right)-\frac{1}{\tilde{k}}\left(\frac{\partial \lambda_{1}}{\partial \nu}\right)\right)-\frac{\lambda_{3}}{2 \lambda_{2}^{3 / 2} \sqrt{n}}\right\}+O\left(\frac{1}{n \sqrt{n}}\right) \\
& =1-\frac{2}{k \lambda_{2} n}\left\{u(\gamma)-\lambda_{1}\right\}\left(\frac{\partial \lambda_{1}}{\partial \gamma}\right)+\frac{2}{\tilde{k} \lambda_{2} n}\left\{u(\nu)-\lambda_{1}\right\}\left(\frac{\partial \lambda_{1}}{\partial \nu}\right)-\frac{3}{\lambda_{2} n}\left\{\frac{1}{k^{2}}\left(\frac{\partial \lambda_{1}}{\partial \gamma}\right)^{2}+\frac{1}{\tilde{k}^{2}}\left(\frac{\partial \lambda_{1}}{\partial \nu}\right)^{2}\right\} \\
& -\frac{2}{\lambda_{2} n}\left\{\frac{1}{k^{2}}\left(\frac{\partial k}{\partial \theta}\right)\left(\frac{\partial \lambda_{1}}{\partial \gamma}\right)-\frac{1}{\tilde{k}^{2}}\left(\frac{\partial \tilde{k}}{\partial \theta}\right)\left(\frac{\partial \lambda_{1}}{\partial \nu}\right)\right\}+\frac{11 \lambda_{3}^{2}}{4 \lambda_{2}^{3} n}-\frac{\lambda_{4}}{\lambda_{2}^{2} n}+O\left(\frac{1}{n \sqrt{n}}\right),
\end{aligned}
$$

hence, by (4.30)

$$
\begin{aligned}
V_{\theta, \gamma, \nu}\left(\hat{U}^{*}\right) & =E_{\theta, \gamma, \nu}\left(\hat{U}^{*^{2}}\right)-\left\{E_{\theta, \gamma, \nu}\left(\hat{U}^{*}\right)\right\}^{2} \\
& =1-\frac{2}{k \lambda_{2} n}\left(\frac{\partial \lambda_{1}}{\partial \gamma}\right)\left\{u(\gamma)-\lambda_{1}\right\}+\frac{2}{\tilde{k} \lambda_{2} n}\left(\frac{\partial \lambda_{1}}{\partial \nu}\right)\left\{u(\nu)-\lambda_{1}\right\}
\end{aligned}
$$




$$
\begin{aligned}
& -\frac{3}{\lambda_{2} n}\left\{\frac{1}{k^{2}}\left(\frac{\partial \lambda_{1}}{\partial \gamma}\right)^{2}+\frac{1}{\tilde{k}^{2}}\left(\frac{\partial \lambda_{1}}{\partial \nu}\right)^{2}\right\}-\frac{2}{\lambda_{2} n}\left\{\frac{1}{k^{2}}\left(\frac{\partial k}{\partial \theta}\right)\left(\frac{\partial \lambda_{1}}{\partial \gamma}\right)-\frac{1}{\tilde{k}^{2}}\left(\frac{\partial \tilde{k}}{\partial \theta}\right)\left(\frac{\partial \lambda_{1}}{\partial \nu}\right)\right\} \\
& +\frac{11 \lambda_{3}^{2}}{4 \lambda_{2}^{3} n}-\frac{\lambda_{4}}{\lambda_{2}^{2} n}-\frac{\lambda_{3}^{2}}{4 \lambda_{2}^{3} n}+O\left(\frac{1}{n \sqrt{n}}\right) \\
=1 & +\frac{1}{n}\left(\frac{5 \lambda_{3}^{2}}{2 \lambda_{2}^{3}}-\frac{\lambda_{4}}{\lambda_{2}^{2}}\right)-\frac{2}{k \lambda_{2} n}\left(\frac{\partial \lambda_{1}}{\partial \gamma}\right)\left\{u(\gamma)-\lambda_{1}+\frac{1}{k}\left(\frac{\partial k}{\partial \theta}\right)\right\} \\
& -\frac{3}{k^{2} \lambda_{2} n}\left(\frac{\partial \lambda_{1}}{\partial \gamma}\right)^{2}+\frac{2}{\tilde{k} \lambda_{2} n}\left(\frac{\partial \lambda_{1}}{\partial \nu}\right)\left\{u(\gamma)-\lambda_{1}+\frac{1}{\tilde{k}}\left(\frac{\partial \tilde{k}}{\partial \theta}\right)\right\} \\
& -\frac{3}{\tilde{k}^{2} \lambda_{2} n}\left(\frac{\partial \lambda_{1}}{\partial \nu}\right)^{2}+O\left(\frac{1}{n^{2}}\right) .
\end{aligned}
$$

Since

$$
\lambda_{1}(\theta, \gamma, \nu)=\frac{\partial}{\partial \theta} \log b(\theta, \gamma, \nu)=\frac{1}{b(\theta, \gamma, \nu)} \int_{\gamma}^{\nu} u(x) a(x) e^{\theta u(x)} d x
$$

it follows that

$$
\begin{aligned}
& \frac{\partial \lambda_{1}(\theta, \gamma, \nu)}{\partial \gamma}=\frac{a(\gamma) e^{\theta u(\gamma)}}{b(\theta, \gamma, \nu)}\left\{\lambda_{1}(\theta, \gamma, \nu)-u(\gamma)\right\}=k(\theta, \gamma, \nu)\left\{\lambda_{1}(\theta, \gamma, \nu)-u(\gamma)\right\} \\
& \frac{\partial \lambda_{1}(\theta, \gamma, \nu)}{\partial \nu}=\frac{a(\nu) e^{\theta u(\nu)}}{b(\theta, \gamma, \nu)}\left\{u(\nu)-\lambda_{1}(\theta, \gamma, \nu)\right\}=\tilde{k}(\theta, \gamma, \nu)\left\{u(\nu)-\lambda_{1}(\theta, \gamma, \nu)\right\}
\end{aligned}
$$

Since

$$
\frac{\partial k}{\partial \theta}(\theta, \gamma, \nu)=k(\theta, \gamma, \nu)\left\{u(\gamma)-\lambda_{1}(\theta, \gamma, \nu)\right\}, \quad \frac{\partial \tilde{k}}{\partial \theta}(\theta, \gamma, \nu)=\tilde{k}(\theta, \gamma, \nu)\left\{u(\nu)-\lambda_{1}(\theta, \gamma, \nu)\right\},
$$

it is seen from (4.31) to (4.33) that

$$
V_{\theta, \gamma, \nu}\left(\hat{U}^{*}\right)=1+\frac{1}{n}\left(\frac{5 \lambda_{3}^{2}}{2 \lambda_{2}^{3}}-\frac{\lambda_{4}}{\lambda_{2}^{2}}\right)+\frac{1}{\lambda_{2} n}\left[\left\{\lambda_{1}-u(\gamma)\right\}^{2}+\left\{\lambda_{1}-u(\nu)\right\}^{2}\right]+O\left(\frac{1}{n \sqrt{n}}\right) .
$$

In a similar way to the case of $U_{\gamma, \nu}$, it is easily seen from the first terms of (4.4), (4.17), (4.26), (4.29), (4.30) and (4.34) that $\hat{U}$ and $\hat{U}^{*}$ are asymptotically normal with mean 0 and variance 1. But, it is noted from (3.8) and (4.34) that there is a difference between $\hat{\theta}_{M L}^{\gamma, \nu}$ and $\hat{\theta}_{M L}^{*}$ in the second order, i.e. the order $n^{-1}$, in their asymptotic variances which is discussed in Section 6 in detail. 


\section{The stochastic expansion and the asymptotic variance of the MCLE $\hat{\theta}_{M C L}$ of $\theta$ when $\gamma$ and $\nu$ are unknown}

Let $Y_{2}, \ldots, Y_{n-1}$ be a random permutation of the $(n-2)$ ! permutations of $X_{(2)}, \ldots, X_{(n-1)}$ such that conditional on $X_{(1)}=x_{(1)}$ and $X_{(n)}=x_{(n)}$, the $Y_{2}, \ldots, Y_{n-1}$ are i.i.d. random variables with a density

$$
f\left(y ; \theta, x_{(1)}, x_{(n)}\right)=\frac{a(y) e^{\theta u(y)}}{b\left(\theta, x_{(1)}, x_{(n)}\right)} \quad \text { for } \quad c<\gamma<x_{(1)}<y<x_{(n)}<\nu<d .
$$

For given $X_{(1)}=x_{(1)}$ and $X_{(n)}=x_{(n)}$, the conditional likelihood function of $\theta$ for $\boldsymbol{y}=\left(y_{2}, \ldots, y_{n-1}\right)$ satisfying $c<\gamma<x_{(1)} \leq y_{i} \leq x_{(n)}<\nu<d(i=2, \ldots, n-1)$ is

$$
L\left(\theta ; \boldsymbol{y} \mid x_{(1)}, x_{(n)}\right)=\frac{1}{b^{n-2}\left(\theta, x_{(1)}, x_{(n)}\right)}\left\{\prod_{i=2}^{n-1} a\left(y_{i}\right)\right\} \exp \left\{\theta \sum_{i=2}^{n-1} u\left(y_{i}\right)\right\} .
$$

Then the likelihood equation is

$$
\frac{1}{n-2} \sum_{i=2}^{n-1} u\left(y_{i}\right)-\lambda_{1}\left(\theta, x_{(1)}, x_{(n)}\right)=0
$$

Since there exists a unique solution of (5.2), we denote it by $\hat{\theta}_{M C L}$, i.e. the value of $\theta$ for which $L\left(\theta ; \boldsymbol{y} \mid x_{(1)}, x_{(n)}\right)$ attains supremum. Since, from (5.2)

$$
\begin{aligned}
0= & \frac{1}{n-2} \sum_{i=2}^{n-1}\left\{u\left(Y_{i}\right)-\lambda_{1}\left(\theta, x_{(1)}, x_{(n)}\right)\right\}-\frac{1}{\sqrt{n}} \lambda_{2}\left(\theta, x_{(1)}, x_{(n)}\right) \sqrt{n}\left(\hat{\theta}_{M C L}-\theta\right) \\
& -\frac{1}{2 n} \lambda_{3}\left(\theta, x_{(1)}, x_{(n)}\right) n\left(\hat{\theta}_{M C L}-\theta\right)^{2}-\frac{1}{6 n \sqrt{n}} \lambda_{4}\left(\theta, x_{(1)}, x_{(n)}\right) n \sqrt{n}\left(\hat{\theta}_{M C L}-\theta\right)^{3}+O_{p}\left(\frac{1}{n^{2}}\right),
\end{aligned}
$$

letting

$$
\begin{aligned}
\tilde{Z}_{1} & :=\frac{1}{\sqrt{\tilde{\lambda}_{2}}(n-2)} \sum_{i=2}^{n-1}\left\{u\left(Y_{i}\right)-\lambda_{1}\left(\theta, x_{(1)}, x_{(n)}\right)\right\}, \\
\tilde{U} & :=\sqrt{\tilde{\lambda}_{2} n}\left(\hat{\theta}_{M C L}-\theta\right),
\end{aligned}
$$

where $\tilde{\lambda}_{i}=\lambda_{i}\left(\theta, x_{(1)}, x_{(n)}\right)(i=1,2,3,4)$, we have

$$
0=\sqrt{\frac{\tilde{\lambda_{2}}}{n-2}} \tilde{Z_{1}}-\sqrt{\frac{\tilde{\lambda_{2}}}{n}} \tilde{U}-\frac{\tilde{\lambda_{3}}}{2 \tilde{\lambda_{2}} n} \tilde{U}^{2}-\frac{\tilde{\lambda_{4}}}{6{\tilde{\lambda_{2}}}^{3 / 2} n \sqrt{n}} \tilde{U}^{3}+O_{p}\left(\frac{1}{n^{2}}\right)
$$


hence the stochastic expansion of $\hat{U}$ is given by

$$
\begin{aligned}
\tilde{U} & =\sqrt{\frac{n}{n-2}} \tilde{Z}_{1}-\frac{\tilde{\lambda_{3}}}{2{\tilde{\lambda_{2}}}^{3 / 2} \sqrt{n}} \tilde{U}^{2}-\frac{\tilde{\lambda}_{4}}{6{\tilde{\lambda_{2}}}^{2} n} \tilde{U}^{3}+O_{p}\left(\frac{1}{n \sqrt{n}}\right) \\
& =\tilde{Z}_{1}-\frac{\tilde{\lambda}_{3}}{2{\tilde{\lambda_{2}}}^{3 / 2} \sqrt{n}}{\tilde{Z_{1}}}^{2}+\frac{1}{n} \tilde{Z}_{1}+\frac{\tilde{\lambda}_{3}^{2}}{2{\tilde{\lambda_{2}}}^{3} n} \tilde{Z}_{1}^{3}-\frac{{\tilde{\lambda_{4}}}^{2}}{6{\tilde{\lambda_{2}}}^{2} n} \tilde{Z}^{3}+O_{p}\left(\frac{1}{n \sqrt{n}}\right) .
\end{aligned}
$$

Since for $i=2,3,4$

$$
\tilde{\lambda}_{i}=\lambda_{i}\left(\theta, X_{(1)}, X_{(n)}\right)=\lambda_{i}(\theta, \gamma, \nu)+\frac{1}{n}\left(\frac{\partial \lambda_{i}}{\partial \gamma}\right) T_{(1)}+\frac{1}{n}\left(\frac{\partial \lambda_{i}}{\partial \nu}\right) T_{(n)}+O_{p}\left(\frac{1}{n^{2}}\right)
$$

we obtain

$$
\begin{aligned}
\tilde{U} & =\sqrt{\tilde{\lambda}_{2} n}\left(\hat{\theta}_{M C L}-\theta\right) \\
& =\sqrt{\lambda_{2}}\left\{1+\frac{1}{n \lambda_{2}}\left(\frac{\partial \lambda_{2}}{\partial \gamma}\right) T_{(1)}+\frac{1}{n \lambda_{2}}\left(\frac{\partial \lambda_{2}}{\partial \nu}\right) T_{(n)}+O_{p}\left(\frac{1}{n^{2}}\right)\right\}^{1 / 2} \sqrt{n}\left(\hat{\theta}_{M C L}-\theta\right) \\
& =\sqrt{\lambda_{2} n}\left(\hat{\theta}_{M C L}-\theta\right)\left\{1+\frac{1}{2 n \lambda_{2}}\left(\frac{\partial \lambda_{2}}{\partial \gamma}\right) T_{(1)}+\frac{1}{2 n \lambda_{2}}\left(\frac{\partial \lambda_{2}}{\partial \nu}\right) T_{(n)}+O_{p}\left(\frac{1}{n^{2}}\right)\right\},
\end{aligned}
$$

where $T_{(1)}=n\left(X_{(1)}-\gamma\right), T_{(n)}=n\left(X_{(n)}-\nu\right)$ and $\lambda_{2}=\lambda_{2}(\theta, \gamma, \nu)$. Then it follows from (5.3) and (5.4) that

$$
\begin{aligned}
\tilde{U}_{0}= & \sqrt{\lambda_{2} n}\left(\hat{\theta}_{M C L}-\theta\right) \\
= & \tilde{Z}_{1}-\frac{\tilde{\lambda}_{3}}{2 \tilde{\lambda}_{2}^{3 / 2} \sqrt{n}} \tilde{Z}_{1}^{2}+\frac{1}{n}\left\{1-\frac{1}{2 \lambda_{2}}\left(\frac{\partial \lambda_{2}}{\partial \gamma}\right) T_{(1)}-\frac{1}{2 \lambda_{2}}\left(\frac{\partial \lambda_{2}}{\partial \nu}\right) T_{(n)}\right\} \tilde{Z}_{1} \\
& +\frac{1}{2 n}\left(\frac{\tilde{\lambda}_{3}^{2}}{\tilde{\lambda}_{2}^{3}}-\frac{\tilde{\lambda}_{4}}{3 \tilde{\lambda}_{2}^{2}}\right) \tilde{Z}_{1}^{3}+O_{p}\left(\frac{1}{n \sqrt{n}}\right) .
\end{aligned}
$$

For given $X_{(1)}=x_{(1)}$ and $X_{(n)}=x_{(n)}$, i.e. $T_{(1)}=t_{(1)}:=n\left(x_{(1)}-\gamma\right)$ and $T_{(n)}=t_{(n)}:=n\left(x_{(n)}-\nu\right)$, the conditional expectation of $\tilde{Z}_{1}$ and $\tilde{Z}_{1}{ }^{2}$ are

$$
\begin{aligned}
E_{\theta, \gamma, \nu}\left(\tilde{Z}_{1} \mid t_{(1)}, t_{(n)}\right) & =\frac{1}{\sqrt{\tilde{\lambda}_{2}(n-2)}} \sum_{i=2}^{n-1}\left\{E_{\theta, \gamma, \nu}\left[u\left(Y_{i}\right) \mid t_{(1)}, t_{(n)}\right]-\lambda_{1}\left(\theta, x_{(1)}, x_{(n)}\right)\right\}=0 \\
E_{\theta, \gamma, \nu}\left(\tilde{Z}_{1}^{2} \mid t_{(1)}, t_{(n)}\right) & =\frac{1}{\tilde{\lambda}_{2}(n-2)}\left[\sum_{i=2}^{n-1} E_{\theta, \gamma, \nu}\left[\left\{u\left(Y_{i}\right)-\lambda_{1}\left(\theta, x_{(1)}, x_{(n)}\right)\right\}^{2} \mid t_{(1)}, t_{(n)}\right]\right. \\
+ & \left.\sum_{\substack{i \neq j \\
2 \leq i, j \leq n-1}} E_{\theta, \gamma, \nu}\left[\left\{u\left(Y_{i}\right)-\lambda_{1}\left(\theta, x_{(1)}, x_{(n)}\right)\right\}\left\{u\left(Y_{j}\right)-\lambda_{1}\left(\theta, x_{(1)}, x_{(n)}\right)\right\} \mid t_{(1)}, t_{(n)}\right]\right] \\
& =1
\end{aligned}
$$


hence the conditional variance of $\tilde{Z}_{1}$ is equal to 1 , i.e. $V_{\theta, \gamma, \nu}\left(\tilde{Z}_{1} \mid t_{(1)}, t_{(n)}\right)=1$. In a similar way to the above, we have

$$
E_{\theta, \gamma, \nu}\left(\tilde{Z}_{1}^{3} \mid t_{(1)}, t_{(n)}\right)=\frac{\tilde{\lambda_{3}}}{{\tilde{\lambda_{2}}}^{3 / 2} \sqrt{n-2}}, \quad E_{\theta, \gamma, \nu}\left(\tilde{Z}_{1}^{4} \mid t_{(1)}, t_{(n)}\right)=3+\frac{\tilde{\lambda_{4}}}{{\tilde{\lambda_{2}}}^{2}(n-2)} .
$$

Then it follows from (5.5) to (5.7) that

$$
\begin{aligned}
& E_{\theta, \gamma, \nu}\left(\tilde{U}_{0} \mid T_{(1)}, T_{(n)}\right)=E_{\theta, \gamma, \nu}\left(\tilde{Z}_{1} \mid T_{(1)}, T_{(n)}\right)-\frac{\tilde{\lambda_{3}}}{2{\tilde{\lambda_{2}}}^{3 / 2} \sqrt{n}} E_{\theta, \gamma, \nu}\left(\tilde{Z}_{1}^{2} \mid T_{(1)}, T_{(n)}\right) \\
& +\frac{1}{n} E_{\theta, \gamma, \nu}\left(\tilde{Z}_{1} \mid T_{(1)}, T_{(n)}\right)-\frac{1}{2 \lambda_{2} n}\left(\frac{\partial \lambda_{2}}{\partial \gamma}\right) T_{(1)} E_{\theta, \gamma, \nu}\left(\tilde{Z}_{1} \mid T_{(1)}, T_{(n)}\right) \\
& -\frac{1}{2 \lambda_{2} n}\left(\frac{\partial \lambda_{2}}{\partial \nu}\right) T_{(n)} E_{\theta, \gamma, \nu}\left(\tilde{Z}_{1} \mid T_{(1)}, T_{(n)}\right) \\
& +\frac{1}{2 n}\left(\frac{{\tilde{\lambda_{3}}}^{2}}{{\tilde{\lambda_{2}}}^{3}}-\frac{\tilde{\lambda_{4}}}{3{\tilde{\lambda_{2}}}^{2}}\right) E_{\theta, \gamma, \nu}\left({\tilde{Z_{1}}}^{3} \mid T_{(1)}, T_{(n)}\right)+O_{p}\left(\frac{1}{n \sqrt{n}}\right) \\
& =-\frac{\tilde{\lambda_{3}}}{2{\tilde{\lambda_{2}}}^{3 / 2} \sqrt{n}}+O_{p}\left(\frac{1}{n \sqrt{n}}\right) \\
& E_{\theta, \gamma, \nu}\left(\tilde{U}_{0}^{2} \mid T_{(1)}, T_{(n)}\right)=E_{\theta, \gamma, \nu}\left(\tilde{Z}_{1}^{2} \mid T_{(1)}, T_{(n)}\right)-\frac{\tilde{\lambda_{3}}}{{\tilde{\lambda_{2}}}^{3 / 2} \sqrt{n}} E_{\theta, \gamma, \nu}\left(\tilde{Z}_{1}^{3} \mid T_{(1)}, T_{(n)}\right) \\
& +\frac{{\tilde{\lambda_{3}}}^{2}}{4{\tilde{\lambda_{2}}}^{3} n} E_{\theta, \gamma, \nu}\left(\tilde{Z}_{1}^{4} \mid T_{(1)}, T_{(n)}\right) \\
& +\frac{2}{n} E_{\theta, \gamma, \nu}\left(\tilde{Z}_{1}^{2} \mid T_{(1)}, T_{(n)}\right)+\frac{1}{n}\left(\frac{\tilde{\lambda}_{3}^{2}}{\tilde{\lambda}_{2}^{3}}-\frac{\tilde{\lambda}_{4}}{3 \tilde{\lambda}_{2}^{2}}\right) E_{\theta, \gamma, \nu}\left(\tilde{Z}_{1}^{4} \mid T_{(1)}, T_{(n)}\right) \\
& -\frac{1}{\lambda_{2} n}\left(\frac{\partial \lambda_{2}}{\partial \gamma}\right) T_{(1)} E_{\theta, \gamma, \nu}\left(\tilde{Z}_{1}^{2} \mid T_{(1)}, T_{(n)}\right) \\
& -\frac{1}{\lambda_{2} n}\left(\frac{\partial \lambda_{2}}{\partial \nu}\right) T_{(n)} E_{\theta, \gamma, \nu}\left(\tilde{Z}_{1}^{2} \mid T_{(1)}, T_{(n)}\right)+O_{p}\left(\frac{1}{n \sqrt{n}}\right) \\
& =1+\frac{2}{n}+\frac{11{\tilde{\lambda_{3}}}^{2}}{4{\tilde{\lambda_{2}}}^{3} n}-\frac{\tilde{\lambda_{4}}}{{\tilde{\lambda_{2}}}^{2} n}-\frac{1}{\lambda_{2} n}\left(\frac{\partial \lambda_{2}}{\partial \gamma}\right) T_{(1)} \\
& -\frac{1}{\lambda_{2} n}\left(\frac{\partial \lambda_{2}}{\partial \nu}\right) T_{(n)}+O_{p}\left(\frac{1}{n \sqrt{n}}\right) \text {, }
\end{aligned}
$$


where $\tilde{\lambda}_{i}=\lambda_{i}\left(\theta, X_{(1)}, X_{(n)}\right)(i=2,3,4)$. Since, for $i=2,3,4$

$$
\begin{aligned}
\tilde{\lambda}_{i} & =\lambda_{i}\left(\theta, X_{(1)}, X_{(n)}\right)=\lambda_{i}(\theta, \gamma, \nu)+\frac{1}{n}\left(\frac{\partial \lambda_{i}}{\partial \gamma}\right) T_{(1)}+\frac{1}{n}\left(\frac{\partial \lambda_{i}}{\partial \nu}\right) T_{(n)}+O_{p}\left(\frac{1}{n^{2}}\right) \\
& =\lambda_{i}(\theta, \gamma, \nu)+O_{p}\left(\frac{1}{n}\right)=\lambda_{i}+O_{p}\left(\frac{1}{n}\right)
\end{aligned}
$$

it follows from (5.8) that

$$
\begin{aligned}
E_{\theta, \gamma, \nu}\left(\tilde{U}_{0}\right) & =E_{\theta, \gamma, \nu}\left[E_{\theta, \gamma, \nu}\left(\tilde{U}_{0} \mid T_{(1)}, T_{(n)}\right)\right]=-\frac{1}{2 \sqrt{n}} E_{\theta, \gamma, \nu}\left(\frac{\tilde{\lambda}_{3}}{{\tilde{\lambda_{2}}}^{3 / 2}}\right)+O\left(\frac{1}{n \sqrt{n}}\right) \\
& =-\frac{\lambda_{3}}{2 \lambda_{2}^{3 / 2} \sqrt{n}}+O\left(\frac{1}{n \sqrt{n}}\right) .
\end{aligned}
$$

It is noted from $(3.7),(4.30)$ and (5.11) that

$$
E_{\theta, \gamma, \nu}\left(U_{\gamma, \nu}\right)=E_{\theta, \gamma, \nu}\left(\hat{U}^{*}\right)=E_{\theta, \gamma, \nu}\left(\tilde{U}_{0}\right)=-\frac{\lambda_{3}}{2 \lambda_{2}^{3 / 2} \sqrt{n}}+O\left(\frac{1}{n \sqrt{n}}\right),
$$

hence, $\hat{\theta}_{M C L}$ has the same asymptotic bias as $\hat{\theta}_{M L}^{\gamma, \nu}$ up to the second order. It is also remarked that $\hat{\theta}_{M C L}$ has an advantage over $\hat{\theta}_{M L}$ in the sense of no need of the bias-adjustment. In a similar way to the above, we obtain from (4.6), (4.8), (5.9) and (5.10) that

$$
E_{\theta, \gamma, \nu}\left(\tilde{U}_{0}^{2}\right)=1+\frac{2}{n}+\frac{11 \lambda_{3}^{2}}{4 \lambda_{2}^{3} n}-\frac{\lambda_{4}}{\lambda_{2}^{2} n}-\frac{1}{k \lambda_{2} n}\left(\frac{\partial \lambda_{2}}{\partial \gamma}\right)+\frac{1}{\tilde{k} \lambda_{2} n}\left(\frac{\partial \lambda_{2}}{\partial \nu}\right)+O\left(\frac{1}{n \sqrt{n}}\right) .
$$

Since, by (4.32) and (4.33)

$$
\begin{aligned}
\frac{1}{k}\left(\frac{\partial \lambda_{2}}{\partial \gamma}\right) & =\frac{1}{k}\left(\frac{\partial^{2} \lambda_{1}}{\partial \theta \partial \gamma}\right)=\frac{1}{k} \frac{\partial}{\partial \theta}\left(\frac{\partial \lambda_{1}}{\partial \gamma}\right)=\frac{1}{k} \frac{\partial}{\partial \theta}\left\{k\left(\lambda_{1}-u(\gamma)\right)\right\} \\
& =\frac{1}{k}\left\{\frac{\partial k}{\partial \theta}\left(\lambda_{1}-u(\gamma)\right)+k\left(\frac{\partial \lambda_{1}}{\partial \theta}\right)\right\}=-\left(\lambda_{1}-u(\gamma)\right)^{2}+\lambda_{2}, \\
\frac{1}{\tilde{k}}\left(\frac{\partial \lambda_{2}}{\partial \nu}\right) & =\frac{1}{\tilde{k}}\left(\frac{\partial^{2} \lambda_{1}}{\partial \theta \partial \nu}\right)=\frac{1}{\tilde{k}} \frac{\partial}{\partial \theta}\left(\frac{\partial \lambda_{1}}{\partial \nu}\right)=\frac{1}{\tilde{k}} \frac{\partial}{\partial \theta}\left\{\tilde{k}\left(u(\nu)-\lambda_{1}\right)\right\} \\
& =\frac{1}{\tilde{k}}\left\{\frac{\partial \tilde{k}}{\partial \theta}\left(u(\nu)-\lambda_{1}\right)-\tilde{k}\left(\frac{\partial \lambda_{1}}{\partial \theta}\right)\right\}=\left(u(\nu)-\lambda_{1}\right)^{2}-\lambda_{2},
\end{aligned}
$$

it follows from (5.12) that

$$
E_{\theta, \gamma, \nu}\left(\tilde{U}_{0}^{2}\right)=1+\frac{11 \lambda_{3}^{2}}{4 \lambda_{2}^{3} n}-\frac{\lambda_{4}}{\lambda_{2}^{2} n}+\frac{1}{\lambda_{2} n}\left[\left\{\lambda_{1}-u(\gamma)\right\}^{2}+\left\{\lambda_{1}-u(\nu)\right\}^{2}\right]+O\left(\frac{1}{n \sqrt{n}}\right),
$$


hence, by (5.11) and (4.34)

$$
V_{\theta, \gamma, \nu}\left(\tilde{U}_{0}\right)=1+\frac{1}{n}\left(\frac{5 \lambda_{3}^{2}}{2 \lambda_{2}^{3}}-\frac{\lambda_{4}}{\lambda_{2}^{2}}\right)+\frac{1}{\lambda_{2} n}\left[\left\{\lambda_{1}-u(\gamma)\right\}^{2}+\left\{\lambda_{1}-u(\nu)\right\}^{2}\right]+O\left(\frac{1}{n \sqrt{n}}\right),
$$

which is asymptotically equal to $V_{\theta, \gamma, \nu}\left(\hat{U}^{*}\right)$ up to the second order, i.e. the order $n^{-1}$ in their asymptotic variances. In a similar way to the case of $U_{\gamma, \nu}$, it is seen from the first terms of (5.5), (5.11) and (5.13) that $\tilde{U}_{0}$ is asymptotically normal with mean 0 and variance 1.

\section{The second order asymptotic comparison among $\hat{\theta}_{M L}^{\gamma, \nu}, \hat{\theta}_{M L}^{*}$ and $\hat{\theta}_{M C L}$}

Summarizing the results in the previous sections, we have the following from (3.4), (3.7), (3.8), (4.4), (4.29), (4.30), (4.34), (5.5), (5.11) and (5.13).

Theorem 6.1. For a two-sided truncated exponential family $\mathcal{P}$ of distributions with density (2.1) with a natural parameter $\theta$ and truncation parameters $\gamma$ and $\nu$, let $\hat{\theta}_{M L}^{\gamma, \nu}, \hat{\theta}_{M L}^{*}$ and $\hat{\theta}_{M C L}$ be the $M L E$ of $\theta$ when $\gamma$ and $\nu$ are known, the bias-adjusted MLE and the MCLE of $\theta$ when $\gamma$ and $\nu$ are unknown. Then their stochastic expansions are given by

$$
\begin{aligned}
U_{\gamma, \nu}= & \sqrt{\lambda_{2} n}\left(\hat{\theta}_{M L}^{\gamma, \nu}-\theta\right) \\
= & Z_{1}-\frac{\lambda_{3}}{2 \lambda_{2}^{3 / 2} \sqrt{n}} Z_{1}^{2}+\frac{1}{2 n}\left(\frac{\lambda_{3}^{2}}{\lambda_{2}^{3}}-\frac{\lambda_{4}}{3 \lambda_{2}^{2}}\right) Z_{1}^{3}+O_{p}\left(\frac{1}{n \sqrt{n}}\right), \\
\hat{U}^{*}= & \sqrt{\lambda_{2} n}\left(\hat{\theta}_{M L}^{*}-\theta\right) \\
= & \hat{U}+\frac{1}{\sqrt{\lambda_{2} n}}\left\{\frac{1}{k}\left(\frac{\partial \lambda_{1}}{\partial \gamma}\right)-\frac{1}{\tilde{k}}\left(\frac{\partial \lambda_{1}}{\partial \nu}\right)\right\} \\
& -\frac{1}{\lambda_{2} n}\left\{\frac{\delta_{1}}{k}-\frac{\delta_{2}}{\tilde{k}}+\frac{1}{k^{2}}\left(\frac{\partial k}{\partial \theta}\right)\left(\frac{\partial \lambda_{1}}{\partial \gamma}\right)-\frac{1}{\tilde{k}^{2}}\left(\frac{\partial \tilde{k}}{\partial \theta}\right)\left(\frac{\partial \lambda_{1}}{\partial \nu}\right)\right\} Z_{1}+O_{p}\left(\frac{1}{n \sqrt{n}}\right), \\
\tilde{U}_{0}= & \sqrt{\lambda_{2} n}\left(\hat{\theta}_{M C L}-\theta\right) \\
= & \tilde{Z}_{1}-\frac{\tilde{\lambda}_{3}}{2 \tilde{\lambda}_{2}^{3 / 2} \sqrt{n}} \tilde{Z}_{1}^{2}+\frac{1}{n}\left\{1-\frac{1}{2 \lambda_{2}}\left(\frac{\partial \lambda_{2}}{\partial \gamma}\right) T_{(1)}-\frac{1}{2 \lambda_{2}}\left(\frac{\partial \lambda_{2}}{\partial \nu}\right) T_{(n)}\right\} \tilde{Z}_{1} \\
& +\frac{1}{2 n}\left(\frac{\tilde{\lambda}_{3}^{2}}{\tilde{\lambda}_{2}^{3}}-\frac{\tilde{\lambda}_{4}}{3 \tilde{\lambda}_{2}^{2}}\right) \tilde{Z}_{1}^{3}+O_{p}\left(\frac{1}{n \sqrt{n}}\right),
\end{aligned}
$$

respectively, where

$$
\delta_{1}=\frac{\lambda_{3}}{\lambda_{2}}\left(\frac{\partial \lambda_{1}}{\partial \gamma}\right)-\frac{\partial \lambda_{2}}{\partial \gamma}, \quad \delta_{2}=\frac{\lambda_{3}}{\lambda_{2}}\left(\frac{\partial \lambda_{1}}{\partial \nu}\right)-\frac{\partial \lambda_{2}}{\partial \nu}
$$




$$
\begin{aligned}
\hat{U}= & \sqrt{\lambda_{2} n}\left(\hat{\theta}_{M L}-\theta\right) \\
= & Z_{1}-\frac{\lambda_{3}}{2 \lambda_{2}^{3 / 2} \sqrt{n}} Z_{1}^{2}-\frac{1}{\sqrt{\lambda_{2} n}}\left\{\left(\frac{\partial \lambda_{1}}{\partial \gamma}\right) T_{(1)}+\left(\frac{\partial \lambda_{1}}{\partial \nu}\right) T_{(n)}\right\}+\frac{1}{\lambda_{2} n} Z_{1}\left\{\delta_{1} T_{(1)}+\delta_{2} T_{(n)}\right\} \\
& +\frac{1}{2 n}\left(\frac{\lambda_{3}^{2}}{\lambda_{2}^{3}}-\frac{\lambda_{4}}{3 \lambda_{2}^{2}}\right) Z_{1}^{3}+O_{p}\left(\frac{1}{n \sqrt{n}}\right) .
\end{aligned}
$$

Further, the secon order asymptotic means of $U_{\gamma, \nu}, \hat{U}^{*}$ and $\tilde{U}_{0}$ are given by

$$
E_{\theta}\left(U_{\gamma, \nu}\right)=E_{\theta, \gamma, \nu}\left(\hat{U}^{*}\right)=E_{\theta, \gamma, \nu}\left(\tilde{U}_{0}\right)=-\frac{\lambda_{3}}{2 \lambda_{2}^{3 / 2} \sqrt{n}}+O\left(\frac{1}{n \sqrt{n}}\right),
$$

and the second order asymptotic variances of $U_{\gamma, \nu}, \hat{U}^{*}$ and $\tilde{U}_{0}$ are also given by

$$
\begin{aligned}
V_{\theta}\left(U_{\gamma, \nu}\right) & =1+\frac{1}{n}\left(\frac{5 \lambda_{3}^{2}}{2 \lambda_{2}^{3}}-\frac{\lambda_{4}}{\lambda_{2}^{2}}\right)+O\left(\frac{1}{n \sqrt{n}}\right) \\
V_{\theta, \gamma, \nu}\left(\hat{U}^{*}\right) & =V_{\theta, \gamma, \nu}\left(\tilde{U}_{0}\right) \\
& =1+\frac{1}{n}\left(\frac{5 \lambda_{3}^{2}}{2 \lambda_{2}^{3}}-\frac{\lambda_{4}}{\lambda_{2}^{2}}\right)+\frac{1}{\lambda_{2} n}\left[\left\{\lambda_{1}-u(\gamma)\right\}^{2}+\left\{\lambda_{1}-u(\nu)\right\}^{2}\right]+O\left(\frac{1}{n \sqrt{n}}\right) .
\end{aligned}
$$

Remark In the second order asymptotic variances of $U_{\gamma, \nu}, \hat{U}^{*}$ and $\tilde{U}_{0}$, the first term of order $1 / n$ results from the regular part of the density (2.1), which coincides with the fact that the distribution with (2.1) is considered to belong to a regular exponential family of distributions when $\gamma$ and $\nu$ are known. The second term of order $1 / n$ in $V_{\theta, \gamma, \nu}\left(\hat{U}^{*}\right)$ and $V_{\theta, \gamma, \nu}\left(\tilde{U}_{0}\right)$ follows from the nonregular (i.e. truncation) part of (2.1) when $\gamma$ and $\nu$ are unknown, which means the ratio of the variance $\lambda_{2}=V_{\theta, \gamma, \nu}(u(X))=E_{\theta, \gamma, \nu}\left[\left\{u(X)-\lambda_{1}\right\}^{2}\right]$ of $u(X)$ to the sum of distances $\left\{\lambda_{1}-u(\gamma)\right\}^{2}$ and $\left\{\lambda_{1}-u(\nu)\right\}^{2}$ from the mean $\lambda_{1}$ of $u(X)$ to $u(x)$ at $x=\gamma$ and $u(x)$ at $x=\nu$, respectively.

Comparing the second order asymptotic variances of $U_{\gamma, \nu}, \hat{U}^{*}$ and $\tilde{U}_{0}$ given in Theorem 6.1, we have the following.

Corollary 6.1. Under the same setup as Theorem 6.1, the bias-adjusted MLE $\hat{\theta}_{M L}^{*}$ and the MCLE $\hat{\theta}_{M C L}$ are second order asymptotically equivalent in the sense that

$$
d_{n}\left(\hat{\theta}_{M L}^{*}, \hat{\theta}_{M C L}\right):=n\left\{V_{\theta, \gamma, \nu}\left(\hat{U}^{*}\right)-V_{\theta, \gamma, \nu}\left(\tilde{U}_{0}\right)\right\}=o(1)
$$

as $n \rightarrow \infty$, and they are second order asymptotically worse than $\hat{\theta}_{M L}^{\gamma, \nu}$ with the second order asymp- 
totic losses of $\hat{\theta}_{M L}^{*}$ and $\hat{\theta}_{M C L}$ relative to $\hat{\theta}_{M L}^{\gamma, \nu}$

$$
\begin{aligned}
& d_{n}\left(\hat{\theta}_{M L}^{*}, \hat{\theta}_{M L}^{\gamma, \nu}\right):=n\left\{V_{\theta, \gamma, \nu}\left(\hat{U}^{*}\right)-V_{\theta}\left(U_{\gamma, \nu}\right)\right\}=\frac{1}{\lambda_{2}}\left[\left\{\lambda_{1}-u(\gamma)\right\}^{2}+\left\{\lambda_{1}-u(\nu)\right\}^{2}\right]+o(1), \\
& d_{n}\left(\hat{\theta}_{M C L}, \hat{\theta}_{M L}^{\gamma, \nu}\right):=n\left\{V_{\theta, \gamma, \nu}\left(\tilde{U}_{0}\right)-V_{\theta}\left(U_{\gamma, \nu}\right)\right\}=\frac{1}{\lambda_{2}}\left[\left\{\lambda_{1}-u(\gamma)\right\}^{2}+\left\{\lambda_{1}-u(\nu)\right\}^{2}\right]+o(1)
\end{aligned}
$$

as $n \rightarrow \infty$, respectively.

The proof is straightforward from Theorem 6.1.

\section{$7 \quad$ Examples}

For a two-sided truncated exponential case, an upper-truncated Pareto case and a two-sided truncated normal case, the second order asymptotic losses of the estimators are given as examples.

Example 7.1 (Two-sided truncated exponential distribution). Let $c=-\infty, d=\infty, a(x) \equiv 1$ and $u(x)=-x$ for $-\infty<\gamma \leq x \leq \nu<\infty$ in the density (2.1). Since $b(\theta, \gamma, \nu)=\left(e^{-\theta \gamma}-e^{-\theta \nu}\right) / \theta$, it follows that $\Theta=(0, \infty)$,

$$
\begin{aligned}
& \lambda_{1}=\frac{\partial}{\partial \theta} \log b(\theta, \gamma, \nu)=\frac{-\gamma e^{-\theta \gamma}+\nu e^{-\theta \nu}}{e^{-\theta \gamma}-e^{-\theta \nu}}-\frac{1}{\theta} \\
& \lambda_{2}=\frac{\partial^{2}}{\partial \theta^{2}} \log b(\theta, \gamma, \nu)=\frac{\gamma^{2} e^{-\theta \gamma}-\nu^{2} e^{-\theta \nu}}{e^{-\theta \gamma}-e^{-\theta \nu}}-\frac{\left(\gamma e^{-\theta \gamma}-\nu e^{-\theta \nu}\right)^{2}}{\left(e^{-\theta \gamma}-e^{-\theta \nu}\right)^{2}}+\frac{1}{\theta^{2}} \\
& k(\theta, \gamma, \nu)=\frac{a(\gamma) e^{\theta u(\gamma)}}{b(\theta, \gamma, \nu)}=\frac{\theta e^{-\theta \gamma}}{e^{-\theta \gamma}-e^{-\theta \nu}}, \\
& \tilde{k}(\theta, \gamma, \nu)=\frac{a(\nu) e^{\theta u(\nu)}}{b(\theta, \gamma, \nu)}=\frac{\theta e^{-\theta \nu}}{e^{-\theta \gamma}-e^{-\theta \nu}} .
\end{aligned}
$$

Then it follows from (3.2), (4.2) and (5.2) that the solutions of $\theta$ of the following equations

$$
\begin{aligned}
& \frac{\gamma e^{-\theta \gamma}-\nu e^{-\theta \nu}}{e^{-\theta \gamma}-e^{-\theta \nu}}+\frac{1}{\theta}=\bar{X}, \\
& \frac{X_{(1)} e^{-\theta X_{(1)}}-X_{(n)} e^{-\theta X_{(n)}}}{e^{-\theta X_{(1)}}-e^{-\theta X_{(n)}}}+\frac{1}{\theta}=\bar{X}, \\
& \frac{X_{(1)} e^{-\theta X_{(1)}}-X_{(n)} e^{-\theta X_{(n)}}}{e^{-\theta X_{(1)}}-e^{-\theta X_{(n)}}}+\frac{1}{\theta}=\frac{1}{n-2} \sum_{i=2}^{n-1} X_{(i)}
\end{aligned}
$$

become $\hat{\theta}_{M L}^{\gamma, \nu}, \hat{\theta}_{M L}$ and $\hat{\theta}_{M C L}$, respectively, where $\bar{X}=(1 / n) \sum_{i=1}^{n} X_{i}$. From (4.27) the bias-adjusted 
MLE is seen to be given by

$$
\hat{\theta}_{M L}^{*}=\hat{\theta}_{M L}+\frac{1}{\hat{\lambda_{2}} n}\left\{\frac{1}{\hat{k}}\left(\frac{\partial \hat{\lambda_{1}}}{\partial \gamma}\right)-\frac{1}{\hat{\tilde{k}}}\left(\frac{\partial \hat{\lambda_{1}}}{\partial \nu}\right)\right\},
$$

where $\hat{\lambda}_{i}=\lambda_{i}\left(\hat{\theta}_{M L}, X_{(1)}, X_{(n)}\right)(i=1,2), \hat{k}=k\left(\hat{\theta}_{M L}, X_{(1)}, X_{(n)}\right), \hat{\tilde{k}}=\tilde{k}\left(\hat{\theta}_{M L}, X_{(1)}, X_{(n)}\right)$, and

$$
\frac{\partial \hat{\lambda_{1}}}{\partial \gamma}=\frac{\partial \lambda_{1}}{\partial \gamma}\left(\hat{\theta}_{M L}, X_{(1)}, X_{(n)}\right), \quad \frac{\partial \hat{\lambda_{1}}}{\partial \nu}=\frac{\partial \lambda_{1}}{\partial \nu}\left(\hat{\theta}_{M L}, X_{(1)}, X_{(n)}\right)
$$

with

$$
\begin{aligned}
& \frac{\partial \lambda_{1}}{\partial \gamma}=\frac{-e^{-\theta \gamma}}{e^{-\theta \gamma}-e^{-\theta \nu}}-\frac{\theta(\gamma-\nu) e^{-\theta(\gamma+\nu)}}{\left(e^{-\theta \gamma}-e^{-\theta \nu}\right)^{2}}, \\
& \frac{\partial \lambda_{1}}{\partial \nu}=\frac{e^{-\theta \nu}}{e^{-\theta \gamma}-e^{-\theta \nu}}+\frac{\theta(\gamma-\nu) e^{-\theta(\gamma+\nu)}}{\left(e^{-\theta \gamma}-e^{-\theta \nu}\right)^{2}} .
\end{aligned}
$$

From (6.3) to (6.5) we obtain the second order asymptotic losses

$$
\begin{aligned}
& d_{n}\left(\hat{\theta}_{M L}^{*}, \hat{\theta}_{M C L}\right)=o(1) \\
& d_{n}\left(\hat{\theta}_{M L}^{*}, \hat{\theta}_{M L}^{\gamma, \nu}\right)=d_{n}\left(\hat{\theta}_{M C L}, \hat{\theta}_{M L}^{\gamma, \nu}\right)=\frac{\left(\frac{1}{\theta}+\frac{(\gamma-\nu) e^{-\theta \gamma}}{e^{-\theta \gamma}-e^{-\theta \nu}}\right)^{2}+\left(\frac{1}{\theta}+\frac{(\gamma-\nu) e^{-\theta \nu}}{e^{-\theta \gamma}-e^{-\theta \nu}}\right)^{2}}{\frac{1}{\theta^{2}}-\frac{(\gamma-\nu)^{2} e^{-\theta(\gamma+\nu)}}{\left(e^{-\theta \gamma}-e^{-\theta \nu}\right)^{2}}}+o(1)
\end{aligned}
$$

as $n \rightarrow \infty$.

When $\theta=\gamma=1$, and $\nu=2,3,5$, the values of the second order asymptotic loss $d_{n}\left(\hat{\theta}_{M L}^{*}, \hat{\theta}_{M L}^{\gamma, \nu}\right)$ of $\hat{\theta}_{M L}^{*}$ relative to $\hat{\theta}_{M L}^{\gamma, \nu}$ are given in Table 1 .

Table 1

The values of the second order asymptotic loss $d_{n}\left(\hat{\theta}_{M L}^{*}, \hat{\theta}_{M L}^{\gamma, \nu}\right)$ of $\hat{\theta}_{M L}^{*}$ relative to $\hat{\theta}_{M L}^{\gamma, \nu}$ for $\theta=\gamma=1$.

\begin{tabular}{c|c}
$\nu$ & The constant term of $d_{n}\left(\hat{\theta}_{M L}^{*}, \hat{\theta}_{M L}^{\gamma, \nu}\right)$ \\
\hline 2 & 6.4725 \\
3 & 7.9582 \\
5 & 14.8146
\end{tabular}

Example 7.2 (Upper-truncated Pareto distribution). For the Pareto distribution with an index parameter $\alpha$ to be estimated and two truncation parameters $\gamma$ and $\nu$ as nuisance parameters, Aban et al. (2006) show the asymptotic normality of the MLEs $\tilde{\alpha}$ and $\hat{\alpha}$ of $\alpha$ in the case when $\gamma$ and $\nu$ are known and the case when $\gamma$ and $\nu$ are unknown, respectively. Although it is noted in Remark 2 of their paper that the asymptotic variance of $\hat{\alpha}$ is not the same as that of $\tilde{\alpha}$, it is seen from (6.1) and (6.2) that $\tilde{\alpha}$ and $\hat{\alpha}$ has the same asymptotic variance in the first order. However, in the second 
order asymptotic comparison, a bias-adjustment of $\hat{\alpha}$ is needed and its second order asymptotic variance is different from that of $\tilde{\alpha}$, as below. Note that $\alpha$ is represented as $\theta$ in this paper. Let $c=0, d=\infty, a(x)=1 / x$ and $u(x)=-\log x$ for $0<\gamma \leq x \leq \nu<\infty$ in the density (2.1). Then

$$
b(\theta, \gamma, \nu)=\frac{1-\left(\frac{\gamma}{\nu}\right)^{\theta}}{\theta \gamma^{\theta}}
$$

for $\theta \in \Theta=(0, \infty)$. Letting $t=\log x, \gamma_{0}=\log \gamma$ and $\nu_{0}=\log \nu$, we see that $(2.1)$ becomes

$$
f\left(t ; \theta, \gamma_{0}, \nu_{0}\right)= \begin{cases}\frac{\theta e^{\theta \gamma_{0}}}{1-e^{-\theta\left(\nu_{0}-\gamma_{0}\right)}} e^{-\theta t} & \text { for } \gamma_{0} \leq t \leq \nu_{0} \\ 0 & \text { otherwise }\end{cases}
$$

Hence the upper-truncated Pareto distribution case is reduced to the two-sided truncated exponential one in Example 7.1. Replacing $\bar{X}$ and $X_{(i)}(i=1, \cdots, n)$ by $\overline{\log X}:=(1 / n) \sum_{i=1}^{n} \log X_{i}$ and $\log X_{(i)}(i=1, \cdots, n)$, respectively, in Example 7.1, we have the second order asymptotic losses

$$
\begin{aligned}
& d_{n}\left(\hat{\theta}_{M L}^{*}, \hat{\theta}_{M C L}\right)=o(1) \\
& d_{n}\left(\hat{\theta}_{M L}^{*}, \hat{\theta}_{M L}^{\gamma, \nu}\right)=d_{n}\left(\hat{\theta}_{M C L}, \hat{\theta}_{M L}^{\gamma, \nu}\right)=\left\{\left(1+\frac{\xi \log \xi}{1-\xi}\right)^{2}+\left(1+\frac{\log \xi}{1-\xi}\right)^{2}\right\} /\left\{1-\frac{\xi(\log \xi)^{2}}{(1-\xi)^{2}}\right\}+o(1)
\end{aligned}
$$

as $n \rightarrow \infty$, where $\xi:=(\gamma / \nu)^{\theta}$.

When $\theta=0.8, \gamma=1$, and $\nu=5,10,15$, the values of the second order asymptotic loss $d_{n}\left(\hat{\theta}_{M L}^{*}, \hat{\theta}_{M L}^{\gamma, \nu}\right)$ of $\hat{\theta}_{M L}^{*}$ relative to $\hat{\theta}_{M L}^{\gamma, \nu}$ are given in Table 2. In Aban et al. (2006), the performance of the MLE is compared with that of the estimators of Hill and Beg when $\theta=0.8, \gamma=1$, and $\nu=10$.

Table 2

The values of the second order asymptotic loss $d_{n}\left(\hat{\theta}_{M L}^{*}, \hat{\theta}_{M L}^{\gamma, \nu}\right)$ of $\hat{\theta}_{M L}^{*}$ relative to $\hat{\theta}_{M L}^{\gamma, \nu}$ for $\theta=0.8$ and $\gamma=1$.

\begin{tabular}{c|c}
$\nu$ & The constant term of $d_{n}\left(\hat{\theta}_{M L}^{*}, \hat{\theta}_{M L}^{\gamma, \nu}\right)$ \\
\hline 5 & 6.7898 \\
10 & 7.6495 \\
15 & 8.3155
\end{tabular}

Example 7.3 (Two-sided truncated normal distribution). Let $c=-\infty, d=\infty, a(x)=e^{-x^{2} / 2}$ and $u(x)=x$ for $-\infty<\gamma \leq x \leq \nu<\infty$ in the density (2.1). Since

$$
b(\theta, \gamma, \nu)=\sqrt{2 \pi} e^{\theta^{2} / 2}\{\Phi(\theta-\gamma)-\Phi(\theta-\nu)\},
$$


it follows that $\Theta=(-\infty, \infty)$,

$$
\begin{aligned}
\lambda_{1}(\theta, \gamma, \nu) & =\theta+\eta_{\gamma-\nu}(\theta-\gamma)+\eta_{\nu-\gamma}(\theta-\nu) \\
\lambda_{2}(\theta, \gamma, \nu) & =1-(\theta-\gamma) \eta_{\gamma-\nu}(\theta-\gamma)-(\theta-\nu) \eta_{\nu-\gamma}(\theta-\nu)-\left\{\eta_{\gamma-\nu}(\theta-\gamma)+\eta_{\nu-\gamma}(\theta-\nu)\right\}^{2} \\
k(\theta, \gamma, \nu) & =\eta_{\gamma-\nu}(\theta-\gamma), \quad \tilde{k}(\theta, \gamma, \nu)=-\eta_{\nu-\gamma}(\theta-\nu)
\end{aligned}
$$

where

$$
\eta_{\alpha}(t):=\frac{\phi(t)}{\Phi(t)-\Phi(t+\alpha)}
$$

with

$$
\Phi(t)=\int_{-\infty}^{t} \phi(x) d x, \quad \phi(x)=\frac{1}{\sqrt{2 \pi}} e^{-x^{2} / 2}
$$

for all $t \in \mathbf{R}^{1}$ and all $x \in \mathbf{R}^{1}$. Then it follows from (3.2), (4.2) and (5.2) that the solutions of the following equations

$$
\begin{aligned}
& \theta+\eta_{\gamma-\nu}(\theta-\gamma)-\eta_{\nu-\gamma}(\theta-\nu)=\bar{X} \\
& \theta+\eta_{X_{(1)}-X_{(n)}}\left(\theta-X_{(1)}\right)-\eta_{X_{(n)}-X_{(1)}}\left(\theta-X_{(n)}\right)=\bar{X}
\end{aligned}
$$

and

$$
\theta-\eta_{X_{(1)}-X_{(n)}}\left(\theta-X_{(1)}\right)-\eta_{X_{(n)}-X_{(1)}}\left(\theta-X_{(n)}\right)=\frac{1}{n-2} \sum_{i=2}^{n-1} X_{(i)}
$$

become $\hat{\theta}_{M L}^{\gamma, \nu}, \hat{\theta}_{M L}$ and $\hat{\theta}_{M C L}$, respectively. From (4.27) the bias-adjusted MLE is seen to be given by

$$
\hat{\theta}_{M L}^{*}=\hat{\theta}_{M L}+\frac{1}{\hat{\lambda}_{2} n}\left\{\frac{1}{\hat{k}}\left(\frac{\partial \hat{\lambda}_{1}}{\partial \gamma}\right)-\frac{1}{\hat{\tilde{k}}}\left(\frac{\partial \hat{\lambda}_{1}}{\partial \nu}\right)\right\}
$$

where

$$
\hat{\lambda}_{i}=\lambda_{i}\left(\hat{\theta}_{M L}, X_{(1)}, X_{(n)}\right)(i=1,2), \quad \hat{k}=k\left(\hat{\theta}_{M L}, X_{(1)}, X_{(n)}\right), \quad \hat{\tilde{k}}=\hat{\tilde{k}}\left(\hat{\theta}_{M L}, X_{(1)}, X_{(n)}\right)
$$

and

$$
\frac{\partial \hat{\lambda}_{1}}{\partial \gamma}=\frac{\partial \lambda_{1}}{\partial \gamma}\left(\hat{\theta}_{M L}, X_{(1)}, X_{(n)}\right), \quad \frac{\partial \hat{\lambda}_{1}}{\partial \nu}=\frac{\partial \lambda_{1}}{\partial \nu}\left(\hat{\theta}_{M L}, X_{(1)}, X_{(n)}\right)
$$


with

$$
\begin{aligned}
& \frac{\partial \lambda_{1}}{\partial \gamma}=\eta_{\gamma-\nu}(\theta-\gamma)\left\{\theta-\gamma-\eta_{\gamma-\nu}(\theta-\gamma)-\eta_{\nu-\gamma}(\theta-\nu)\right\} \\
& \frac{\partial \lambda_{1}}{\partial \nu}=\eta_{\nu-\gamma}(\theta-\nu)\left\{\theta-\nu+\eta_{\gamma-\nu}(\theta-\gamma)+\eta_{\nu-\gamma}(\theta-\nu)\right\}
\end{aligned}
$$

From (6.3) to (6.5) we obtain the second order asymptotic losses

$$
\begin{aligned}
d_{n}\left(\hat{\theta}_{M L}^{*}, \hat{\theta}_{M C L}\right) & =o(1) \\
d_{n}\left(\hat{\theta}_{M L}^{*}, \hat{\theta}_{M L}^{\gamma, \nu}\right)= & d_{n}\left(\hat{\theta}_{M C L}, \hat{\theta}_{M L}^{\gamma, \nu}\right) \\
= & \frac{\left\{\theta-\gamma+\eta_{\gamma-\nu}(\theta-\gamma)+\eta_{\nu-\gamma}(\theta-\nu)\right\}^{2}+\left\{\theta-\nu+\eta_{\gamma-\nu}(\theta-\gamma)+\eta_{\nu-\gamma}(\theta-\nu)\right\}^{2}}{1-(\theta-\gamma) \eta_{\gamma-\nu}(\theta-\gamma)-(\theta-\nu) \eta_{\nu-\gamma}(\theta-\nu)-\left\{\eta_{\gamma-\nu}(\theta-\gamma)+\eta_{\nu-\gamma}(\theta-\nu)\right\}^{2}}+o(1)
\end{aligned}
$$

as $n \rightarrow \infty$.

When $\theta=\gamma=0$, and $\nu=1,2,3$, the values of the second order asymptotic loss $d_{n}\left(\hat{\theta}_{M L}^{*}, \hat{\theta}_{M L}^{\gamma, \nu}\right)$ of $\hat{\theta}_{M L}^{*}$ relative to $\hat{\theta}_{M L}^{\gamma, \nu}$ are given in Table 3 .

Table 3

The values of the second order asymptotic loss $d_{n}\left(\hat{\theta}_{M L}^{*}, \hat{\theta}_{M L}^{\gamma, \nu}\right)$ of $\hat{\theta}_{M L}^{*}$ relative to $\hat{\theta}_{M L}^{\gamma, \nu}$ for $\theta=\gamma=0$.

\begin{tabular}{c|c}
$\nu$ & The constant term of $d_{n}\left(\hat{\theta}_{M L}^{*}, \hat{\theta}_{M L}^{\gamma, \nu}\right)$ \\
\hline 1 & 6.3154 \\
2 & 8.5681 \\
3 & 15.8437
\end{tabular}

\section{Concluding remarks}

For a one-sided truncated exponential family of distributions with a natural parameter $\theta$ and a truncation parameter $\gamma$ as a nuisance parameter, Akahira (2013) derives the stochastic expansions of the MLE $\hat{\theta}_{M L}^{\gamma}$ of $\theta$ for known $\gamma$, the MLE $\hat{\theta}_{M L}$ and the MCLE $\hat{\theta}_{M C L}$ of $\theta$ for unknown $\gamma$, and shows that a bias-adjusted MLE $\hat{\theta}_{M L}^{*}$ with the same asymptotic bias as $\hat{\theta}_{M L}^{\gamma}$ and $\hat{\theta}_{M C L}$ are second order asymptotically equivalent in the sense that their asymptotic variances are same up to the order $o(1 / n)$ and they are second order asymptotically worse than $\hat{\theta}_{M L}^{\gamma}$ in the second order asymptotic variance. It is easily seen that the same asymptotic normality of $\hat{\theta}_{M L}^{\gamma}, \hat{\theta}_{M L}$ and $\hat{\theta}_{M C L}$ results from the first term of their stochastic expansions. The second order asymptotic losses of $\hat{\theta}_{M L}^{*}$ and $\hat{\theta}_{M C L}$ relative to $\hat{\theta}_{M L}^{\gamma}$ are also obtained from their second order asymptotic variances. In this paper, in a similar way to Akahira (2013), the above results are extended to the two-sided truncated exponential family of distributions with a natural parameter $\theta$ and two truncation parameters $\gamma$ 
and $\nu$ as nuisance ones, including the upper-truncated Pareto distribution which is important in applications to finance, hydrology and atmospheric science as is seen in Aban et al. (2006). In particular, the second order asymptotic losses of $\hat{\theta}_{M L}^{*}$ and $\hat{\theta}_{M C L}$ given by (6.4) and (6.5) are seen to be quite simple, which results from the two-sided truncated exponential family of distributions. Indeed, as is seen from the form (2.1) of density, (6.1) and (6.2), the structure of the regular and non-regular parts of (2.1) reflects in that of the second order asymptotic variances (6.1) and (6.2) of $U_{\gamma, \nu}=\sqrt{\lambda_{2} n}\left(\hat{\theta}_{M L}^{\gamma, \nu}-\theta\right), \hat{U}^{*}=\sqrt{\lambda_{2} n}\left(\hat{\theta}_{M L}^{*}-\theta\right)$ and $\tilde{U}_{0}=\sqrt{\lambda_{2} n}\left(\hat{\theta}_{M C L}-\theta\right)$. The regular part corresponds to the term of order $n^{-1}$ in the second order asymptotic variance (6.1) of $U_{\gamma, \nu}$, where $\gamma$ and $\nu$ are known. When $\gamma$ and $\nu$ are unknown, the second order asymptotic variances of $\hat{U}^{*}$ and $\tilde{U}_{0}$ consist of the corresponding regular term and the non-regular one with the term depending on $u(\gamma)$ and $u(\nu)$ in the second order, i.e. the order $n^{-1}$ in (6.2). The results arise from giving full consideration to the typical non-regular case up to the second order. Furthermore, in a similar way to the above, the results may be extended to the case of a more general truncated family of distributions.

\section{Acknowledgements}

The authors wish to thank the referee for kind comments.

\section{References}

[1] Aban, I. B., Meerschaert, M. M. and Panorska, A. K. (2006). Parameter estimation for the truncated Pareto distribution. Journal of the American Statistical Association, 101, 270-277.

[2] Akahira, M. (1981). On asymptotic deficiency of estimators. Australian Journal of Statistics, $23,67-72$.

[3] Akahira, M. (1986). The Structure of Asymptotic Deficiency of Estimators. Queen's Papers in Pure and Applied Mathematics, 75, Queen's University Press, Kingston, Canada.

[4] Akahira, M. (1992). Higher order asymptotics and asymptotic deficiency of estimators. Selecta Statistica Canadiana, 8, 1-36. 
[5] Akahira, M. (1997). An information inequality bound for the asymptotic variance of sequential estimation procedures of a linearly combined parameter and its attainment. Sequential Analysis, 16, 47-63.

[6] Akahira, M. (2013). Second order asymptotic comparison of the MLE and MCLE of a natural parameter for a truncated exponential family of distributions. Mathematical Research Note 2013-001, Institute of Mathematics, University of Tsukuba. To appear in Annals of the Institute of Statistical Mathematics.

[7] Akahira, M. and Ohyauchi, N. (2012). The asymptotic expansion of the maximum likelihood estimator for a truncated exponential family of distributions. (In Japanese). RIMS (Research Institute for Mathematical Sciences, Kyoto University) Kôkyûroku, 1804, 188-192.

[8] Akahira, M. and Takeuchi, K (1982). On asymptotic deficiency of estimators in pooled samples in the presence of nuisance parameters. Statistics \& Decisions, 1, 17-38.

[9] Akahira, M. and Takeuchi, K (1991). Second order asymptotic efficiency in terms of the asymptotic variance of sequential estimation procedures in the presence of nuisance parameters. Sequential Analysis, 10, 27-43.

[10] Andersen, E. B. (1970). Asymptotic properties of conditional maximum likelihood estimators. Journal of the Royal Statistical Society, Series B, 32, 283-301.

[11] Bar-Lev, S. K. (1984). Large sample properties of the MLE and MCLE for the natural parameter of a truncated exponential family. Annals of the Institute of Statistical Mathematics, 36, Part A, 217-222.

[12] Barndorff-Nielsen, O. (1978). Information and Exponential Families in Statistical Theory. Wiley, New York.

[13] Hodges, J. L. and Lehmann, E. L. (1970). Deficiency. Annals of Mathematical Statistics, 41, $783-801$.

[14] Quesenberry, C. P. (1975). Transforming samples from truncation parameter distributions to uniformity. Communications in Statistics, 4, 1149-1155. 